\title{
Search for gamma-ray burst classes with the RHESSI satellite
}

\author{
J. Řípa ${ }^{1}$, A. Mészáros ${ }^{1}$, C. Wigger ${ }^{2,4}$, D. Huja ${ }^{1}$, R. Hudec ${ }^{3}$, and W. Hajdas ${ }^{2}$ \\ ${ }^{1}$ Charles University, Faculty of Mathematics and Physics, Astronomical Institute, V Holešovičkách 2, 18000 Prague 8, \\ Czech Republic \\ e-mail: ripa@sirrah.troja.mff.cuni.cz; meszaros@cesnet.cz; David.HUJA@seznam.cz \\ 2 Paul Scherrer Institute, 5232 Villigen, Switzerland \\ e-mail: wojtek.hajdas@psi.ch; claudia.wigger@kanti-wohlen.ch \\ 3 Astronomical Institute, Academy of Sciences of the Czech Republic, 25165 Ondřejov, Czech Republic \\ e-mail: rhudec@asu.cas.cz \\ 4 Kantonsschule Wohlen, 5610 Wohlen, Switzerland \\ Received 4 September 2008 / Accepted 2 February 2009
}

ABSTRACT

\begin{abstract}
Aims. We study a sample of 427 gamma-ray bursts (GRBs), measured by the RHESSI satellite, statistically with respect to duration and hardness ratio.

Methods. Standard statistical tests were used, such as $\chi^{2}$, F-test, and the maximum likelihood ratio test, to compare the number of GRB groups in the RHESSI database with that of the BATSE database.

Results. Previous studies based on the BATSE Catalog claim the existence of an intermediate GRB group, besides the long and short groups. Using only the GRB duration $T_{90}$ as information and $\chi^{2}$ or F-test, we do not find any statistically significant intermediate group in the RHESSI data. However, maximum likelihood ratio test reveals a significant intermediate group. Using the 2-dimensional hardness $/ T_{90}$ plane, the maximum likelihood analysis also reveals a significant intermediate group. In contrast to the BATSE database, the intermediate group in the RHESSI data set is harder than the long one.

Conclusions. The existence of an intermediate group follows not only from the BATSE data set, but also from the RHESSI one.
\end{abstract}

Key words. gamma rays: bursts

\section{Introduction}

In the years 1991-2000, approximately 3000 gamma-ray bursts (GRBs) were detected by the BATSE instrument onboard the Compton Gamma-Ray Observatory (Meegan et al. 2001). After the end of this mission (June 2000), the frequency of GRB detections decreased (to $100-200$ GRBs annually) due to different observational methods employed by the operating satellites. Any observational GRB database, compiled since the year 2000 and later, can therefore be of great significance, such as the GRB observations completed by the RHESSI satellite (Holman et al. 2008) 70/year.

Originally it was found (Kouveliotou et al. 1993) that two GRB classes exist: a short one of duration $\$ 2 \mathrm{~s}$ and a long one of duration $z 2 \mathrm{~s}$. This was confirmed by GRB data from the Konus-Wind instrument (Aptekar et al. 1998). However, some articles point to the existence of three classes of GRBs in the BATSE database with respect to their durations (Horváth 1998, 2002). By applying a maximum likelihood ratio test to durations, the work of Horváth et al. (2008) indicates that there is a statistically significant intermediate group in the Swift data set. Horváth et al. (2004) and Horváth et al. (2006) also claimed that, when using a 2-dimensional plane of hardness ratio versus duration, three classes of GRBs can be found in the BATSE data set. Mukherjee et al. (1998) pointed to the existence of three GRB classes in multiparameter space. In another multidimensional analysis of the BATSE catalog by Chattopadhyay et al. (2007), it was argued that at least three clusters of GRBs are found. Some articles also say that the third class (of intermediate duration), observed by BATSE, is a bias caused by an instrumental effect (Hakkila et al. 2000). In Hakkila et al. (2004), there is a review and discussion of GRB classification, based on statistical clustering and data-mining techniques, placing the intermediate group as a separate source population in doubt.

The purpose of this paper is to investigate the number of GRB groups in another data set, namely in the GRB data set provided by the RHESSI satellite. Although the main goal of the RHESSI satellite is the study of solar physics, it also contains a useful set of GRB observations covering the period 2002-2004. Hence, its study can be maximally useful. Trivially, any comparison of different catalogs using different instruments is useful for an independent confirmation of previous results.

In the first step, we analyse the 1-dimensional duration distribution of GRBs observed by RHESSI, and in the second step, we use the two-dimensional plane of hardness ratio versus duration. To determine the number of GRB groups, standard statistical tests described in Trumpler \& Weaver (1953), Press et al. (1992), and Zey et al. (2006) are used.

The paper is organized as follows. In Sect. 2, the RHESSI satellite and the analysed data set are described. In Sect. 3, we present the duration distribution for the RHESSI GRBs and its analysis. In Sect. 4, the 2-dimensional hardness ratio versus duration distribution and the maximum likelihood fit of these data are presented. In Sects. 5 and 6, we present our discussion and conclusions. At the end of our paper, we list the RHESSI data sample.

\section{The RHESSI data sample}

The Ramaty High Energy Solar Spectroscopic Imager (RHESSI) is a NASA Small Explorer satellite designed to study hard 
X-rays and gamma-rays from solar flares (Lin et al. 2002). It consists mainly of an imaging tube and a spectrometer. The spectrometer consists of nine germanium detectors $(7.1 \mathrm{~cm}$ in diameter with a height of $8.5 \mathrm{~cm}$ ) (Smith et al. 2002). They are only lightly shielded, ensuring that RHESSI is also useful for detecting non-solar photons from any direction (Smith et al. 2003). The energy range for GRB detection extends from about $30 \mathrm{keV}$ to $17 \mathrm{MeV}$. For a wide range of energies and GRB incoming directions, the effective area is around $150 \mathrm{~cm}^{2}$ (Wigger et al. 2006b). With a field of view of about half of the sky, RHESSI observes about one or two GRBs per week. Photon hits are stored event-by-event in onboard memory with a time sampling of $\Delta t=1 \mu \mathrm{s}$ resolution. The energy resolution for lines is excellent at $\Delta E=3 \mathrm{keV}$ for $1000 \mathrm{keV}$.

We used the RHESSI GRB Catalog (Wigger et al. 2008) and the Cosmic Burst List (Hurley 2008) to detect 487 GRBs in the RHESSI data between 2002 February 14 and 2008 April 25. We should describe the strategy by which RHESSI GRBs were found. There is no automatic GRB search routine. Only if there is a message from any other instrument of the IPN (Hurley 2007), the RHESSI data are searched for a GRB signal. Therefore, in our data set there are only GRBs, which are also observed by other instruments. The biggest overlap is with Konus-W. About $85 \%$ of all RHESSI GRBs are also observed by Konus-W (Wigger et al. 2006a).

For a deeper analysis, we chose a subset of 427 GRBs with data of a signal/noise ratio higher than 6 . We used the SolarSoftWare (Freeland et al. 2008) program developed in the Interactive Data Language (RSI IDL) programming application as well as our own IDL routines to derive count light curves (with a time resolution higher than $10 \%$ of the burst's duration for the vast majority of our entire data set) and count fluences from the rear detectors' segments (except number R2) of the RHESSI spectrometer (Smith et al. 2002) in the energy band from $25 \mathrm{keV}$ to $1.5 \mathrm{MeV}$. This data set (and the time resolutions of derived light curves) are listed in Table 7.

\section{Duration distribution}

First, we study the one-dimensional duration distribution. We use $T_{90}$ as the GRB duration, i.e., the time interval during which the cumulative counts increase from $5 \%$ to $95 \%$ above background (Meegan et al. 2001). The $T_{90}$ uncertainty consists of two components. We make an assumption that one is given by the count fluence uncertainty during $T_{90}\left(\delta t_{\mathrm{S}}\right)$, which is given by Poissonian noise, and the second one is the time resolution of derived light curves ( $\left.\delta t_{\text {res }}\right)$. The total $T_{90}$ uncertainty $\delta t$ was calculated to be $\delta t=\sqrt{\delta t_{\mathrm{s}}^{2}+\delta t_{\text {res }}^{2}}$.

The histogram of the times $T_{90}$ is a distribution with two maxima at approximately $0.2 \mathrm{~s}$ and $20 \mathrm{~s}$ (Fig. 1). The histogram consists of 19 equally wide bins on a logarithmic scale (of base 10) starting at $0.09 \mathrm{~s}$ and ending at $273.4 \mathrm{~s}$.

We follow the method completed by Horváth (1998) and fitted one, two (Fig. 1), and three (Fig. 2) log-normal functions and used the $\chi^{2}$ test to evaluate these fits. The minimal number of GRBs per bin is 4 (last bin), and hence the use of the $\chi^{2}$ test is possible.

In the case of the fit with one log-normal function, we obtained $\chi^{2} \simeq 157$ for 17 degrees of freedom (d.o.f.). Therefore, this hypothesis is rejected on a smaller than $0.01 \%$ significance level.

The fit with two log-normal functions is shown in Fig. 1 and the fit with three log-normal functions in Fig. 2. The parameters

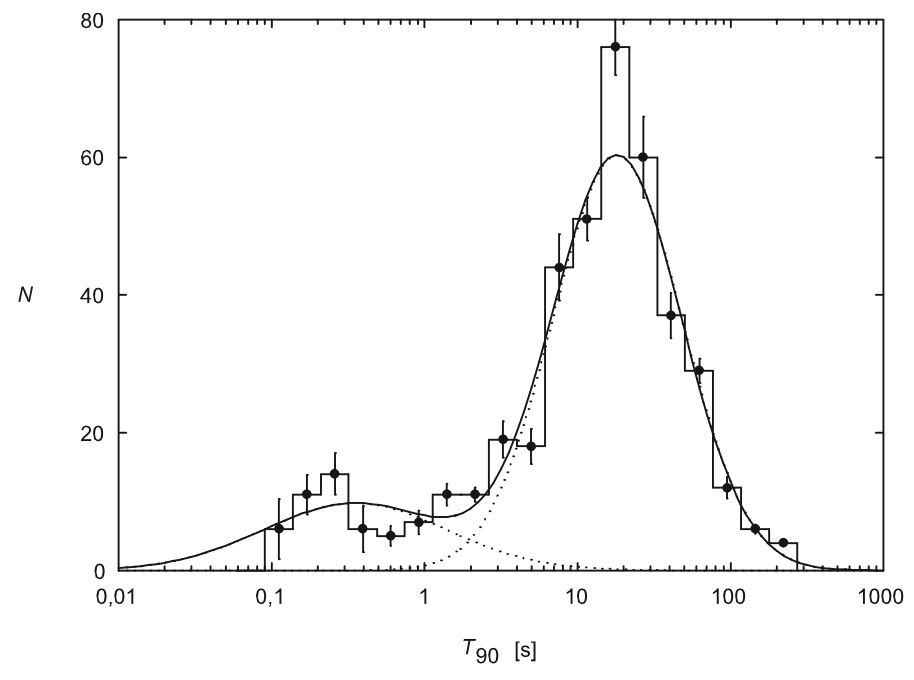

Fig. 1. Duration distribution of the 427 RHESSI bursts with the best $\chi^{2}$ fit of two log-normal functions. Number of bins is 19, d.o.f. $=14$ and $\chi^{2} \simeq 19.1$ which implies the goodness-of-fit $\simeq 16 \%$. The bar errors are standard deviations of the number of GRBs per bin for ten different simulated duration distributions as described in the text.

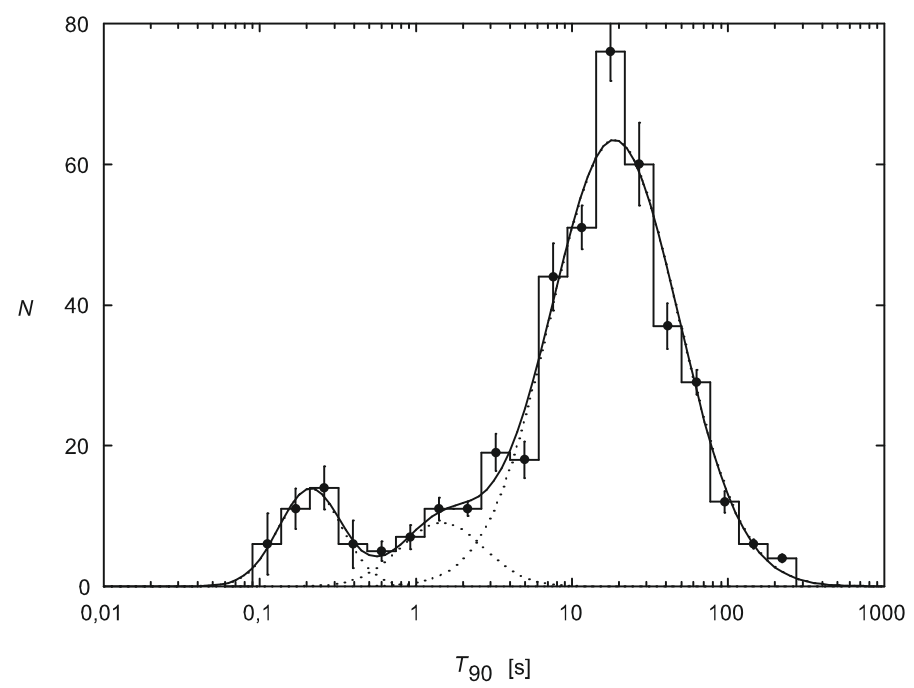

Fig. 2. Duration distribution of the 427 RHESSI bursts with the best $\chi^{2}$ fit of three log-normal functions. Number of bins is 19 , d.o.f. $=11$ and $\chi^{2} \simeq 10.3$ which implies the goodness-of-fit $\simeq 50 \%$. The bar errors are the same as described in Fig. 1.

of the fits, the values of $\chi^{2}$, the degrees of freedom, and the goodness-of-fits are listed in Table 1.

The assumption of two groups being represented by two lognormal fits is acceptable, the fit with three log-normal functions even more. The question is whether the improvement in $\chi^{2}$ is statistically significant. To answer this question, we used the F-test, as described by Band et al. (1997), in their Appendix A. The F-test gives a probability of $6.9 \%$ of the improvement in $\chi^{2}$ being accidental. This value is remarkably low, but not low enough to reject the hypothesis that two log-normal functions are still enough to describe the observed duration distribution.

To determine how the $T_{90}$ uncertainties affect our result, we randomly selected one half of the bursts and shifted their durations by the full amount of their uncertainties to lower values and the second half to higher values. We then compiled a histogram and recalculated the best-fit model parameters, $\chi^{2}$, and F-test. The results for ten such calculations are listed in Table 2. 
Table 1. Parameters of the best $\chi^{2}$ fits of two and three log-normal functions on the RHESSI GRB $T_{90}$ distribution. $\mu$ are the means, $\sigma$ are the standard deviations and $w$ are the weights of the distribution. Given uncertainties are standard deviations of the parameters obtained by ten different fittings of randomly changed histogram of durations by their uncertainties.

\begin{tabular}{lrr}
\hline \hline Parameter & 2 log-normal & 3 log-normal \\
\hline$\mu_{\text {short }}$ & $-0.46 \pm 0.13$ & $-0.68 \pm 0.09$ \\
$\sigma_{\text {short }}$ & $0.60 \pm 0.06$ & $0.20 \pm 0.03$ \\
$w_{\text {short }}[\%]$ & $18.7 \pm 1.5$ & $8.9 \pm 1.0$ \\
$\mu_{\text {long }}$ & $1.26 \pm 0.03$ & $1.27 \pm 0.03$ \\
$\sigma_{\text {long }}$ & $0.42 \pm 0.01$ & $0.41 \pm 0.01$ \\
$w_{\text {long }}[\%]$ & $81.3 \pm 1.5$ & $83.4 \pm 0.9$ \\
$\mu_{\text {middle }}$ & & $0.17 \pm 0.06$ \\
$\sigma_{\text {middle }}$ & & $0.27 \pm 0.06$ \\
$w_{\text {middle }}[\%]$ & & $7.7 \pm 1.0$ \\
d.o.f. & 14 & 11 \\
$\chi^{2}$ & 19.13 & 10.30 \\
goodness[\%] & 16.0 & 50.4 \\
$F_{0}$ & 3.14 & \\
$P\left(F>F_{0}\right)[\%]$ & 6.9 & \\
\hline
\end{tabular}

This method also gives us information about how the fitted parameters vary, and thus informs us of their uncertainties. From Table 2, we see that, on average, the improvement in $\chi^{2}$ is insignificant. Therefore, we cannot proclaim acceptance of three groups by using this statistical method.

Since the number of GRBs is low for many bins, we also used the maximum likelihood method (see Horváth 2002, and the references therein) to fit two and three log-normal functions to the RHESSI data set. The parameters are listed in Table 3.

Since the difference of the logarithms of the likelihoods $\Delta \ln L=9.2$ should be half of the $\chi^{2}$ distribution for 3 degrees of freedom (Horváth 2002), we infer that the introduction of a third group is statistically significant on the $0.036 \%$ level (of being accidental).

To derive an image of how the $T_{90}$ uncertainties affect our result, we proceed as in the $\chi^{2}$ fitting and generate ten different data sets randomly changed in durations by the full amount of their uncertainties. The results are presented in Table 4. From this table, it can be seen that all ten simulations give probabilities, of introducing a third group being accidental, that are much lower than 5\%. Thus, the hypothesis of introducing a third group is highly acceptable.

\section{Hardness ratio versus duration}

Two-dimensional scatter plots of RHESSI GRBs are shown in Figs. 3 and 4 . One axis is the duration $T_{90}$, used in the previous section, the other axis is a hardness ratio. The hardness ratio is defined to be the ratio of two fluences $F$ in two different energy bands integrated over the time interval $T_{90}$. For the RHESSI data set, we used the energy bands (25-120) keV and (120-1500) keV, i.e., $H=F_{120-1500} / F_{25-120}$.

Using the maximum likelihood method (see Horváth et al. 2004, 2006 and the references therein), we fit two and three bivariate log-normal functions to search for clusters. In Fig. 3., we show the best-fit solution of two bivariate log-normal functions (11 independent parameters, since the two weights must add up to $100 \%)$.
Table 2. The minimal $\chi^{2}$, corresponding goodness-of-fits and F-tests for fitted two and three log-normal functions on the RHESSI GRB $T_{90}$ distribution for ten different changes of durations by their uncertainties.

\begin{tabular}{rrrrr}
\hline \hline & 2 log-normal & & 3 log-normal & F-test \\
\hline$\chi^{2}$ & goodness & $\chi^{2}$ & goodness & $P\left(F>F_{0}\right)$ \\
& {$[\%]$} & & {$[\%]$} & {$[\%]$} \\
\hline 23.97 & 4.6 & 14.19 & 22.3 & 11.1 \\
18.03 & 20.6 & 10.65 & 47.3 & 11.0 \\
15.99 & 31.4 & 5.13 & 92.5 & 0.5 \\
13.52 & 48.6 & 7.50 & 75.8 & 8.0 \\
17.87 & 21.3 & 7.55 & 75.3 & 2.0 \\
16.36 & 29.2 & 9.66 & 56.1 & 11.0 \\
11.89 & 61.5 & 6.95 & 80.3 & 10.4 \\
21.86 & 8.2 & 13.77 & 24.6 & 15.1 \\
20.07 & 12.8 & 9.49 & 57.7 & 3.5 \\
20.40 & 11.8 & 12.73 & 31.1 & 14.4 \\
\hline
\end{tabular}

Table 3. Parameters of the best fit with two and three log-normal functions done by the maximum likelihood method on the RHESSI data. $\mu$ are the means, $\sigma$ are the standard deviations, $w$ are the weights of the distribution and $L_{2}, L_{3}$ are the likelihoods. Given uncertainties are standard deviations of the parameters obtained by ten different fittings of data sets, in which the durations were randomly changed by their uncertainties.

\begin{tabular}{lrr}
\hline \hline Parameter & 2 log-normal & 3 log-normal \\
\hline$\mu_{\text {short }}$ & $-0.60 \pm 0.07$ & $-0.64 \pm 0.02$ \\
$\sigma_{\text {short }}$ & $0.25 \pm 0.05$ & $0.20 \pm 0.02$ \\
$w_{\text {short }}[\%]$ & $10.2 \pm 1.3$ & $9.4 \pm 0.4$ \\
$\mu_{\text {long }}$ & $1.20 \pm 0.01$ & $1.26 \pm 0.01$ \\
$\sigma_{\text {long }}$ & $0.47 \pm 0.01$ & $0.41 \pm 0.01$ \\
$w_{\text {long }}[\%]$ & $89.8 \pm 1.3$ & $84.4 \pm 1.0$ \\
$\mu_{\text {middle }}$ & & $0.17 \pm 0.04$ \\
$\sigma_{\text {middle }}$ & & $0.22 \pm 0.06$ \\
$w_{\text {middle }}[\%]$ & & $6.2 \pm 1.4$ \\
$\ln L_{2}$ & -389.17 & \\
$\ln L_{3}$ & & -379.95 \\
\hline
\end{tabular}

The parameters are listed in Table 5. One result is that the short GRBs are on average harder than long GRBs. After a closer look at the GRB distribution within the short class, one can see that the points within the $1 \sigma$ ellipse are not evenly distributed. They cluster towards the shortest durations (Fig. 3).

The fitting of the sum of three groups (17 independent parameters) is shown in Fig. 4. The best-fit model parameters are listed in Table 5. The former short group is clearly separated into two parts. As far as one can tell by sight, the data points scatter evenly within (and around) the $1 \sigma$ ellipses.

Since the difference in the logarithms of the likelihoods $\Delta \ln L=10.9$ should equal one half of the $\chi^{2}$ distribution for 6 degrees of freedom (Horváth et al. 2006), we find that the introduction of a third group is statistically significant at the $0.13 \%$ level (of being accidental).

To derive an image of how GRB durations and hardness ratio uncertainties effect our result, we proceed similarly as in the $\chi^{2}$ fitting and generated ten different data sets randomly changed in duration and hardness ratio by the full amount of their uncertainties. The results are presented in Table 6. From this table, it is seen that almost all of the simulations infer probabilities, of 
Table 4. The maximal likelihoods and corresponding probabilities that introducing of the third group is accidental for maximum likelihood fittings (one-dimensional) with two and three log-normal functions of ten different changes of durations by their uncertainties.

\begin{tabular}{ccc}
\hline \hline $\ln L_{2}$ & $\ln L_{3}$ & $\begin{array}{c}\text { Probability } \\
{[\%]}\end{array}$ \\
\hline-388.12 & -378.86 & 0.03 \\
-390.82 & -383.92 & 0.32 \\
-391.90 & -380.97 & 0.01 \\
-391.75 & -385.37 & 0.52 \\
-392.25 & -384.24 & 0.11 \\
-390.62 & -383.67 & 0.30 \\
-386.26 & -375.54 & 0.01 \\
-392.33 & -384.97 & 0.21 \\
-389.16 & -380.93 & 0.09 \\
-390.21 & -384.32 & 0.82 \\
\hline
\end{tabular}

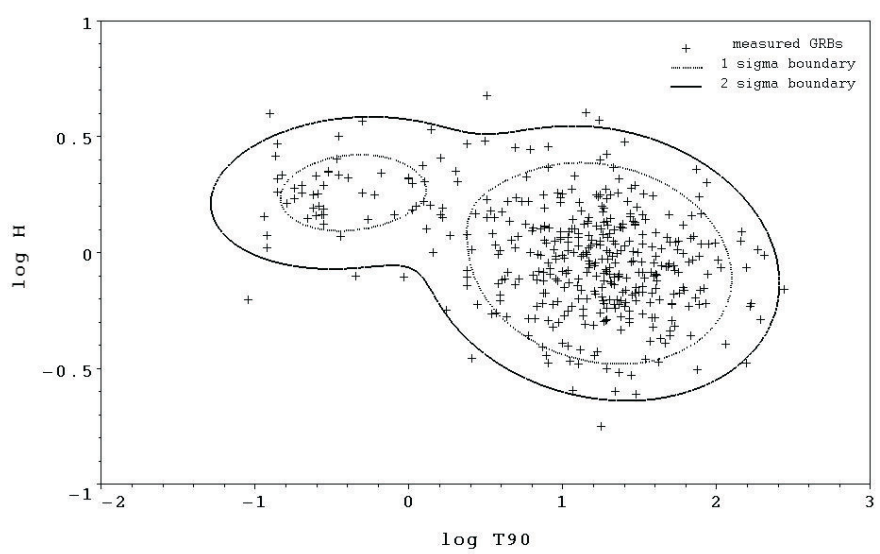

Fig. 3. Hardness ratio vs. $T_{90}$ of the RHESSI GRBs with the best fit of two bivariate log-normal functions.

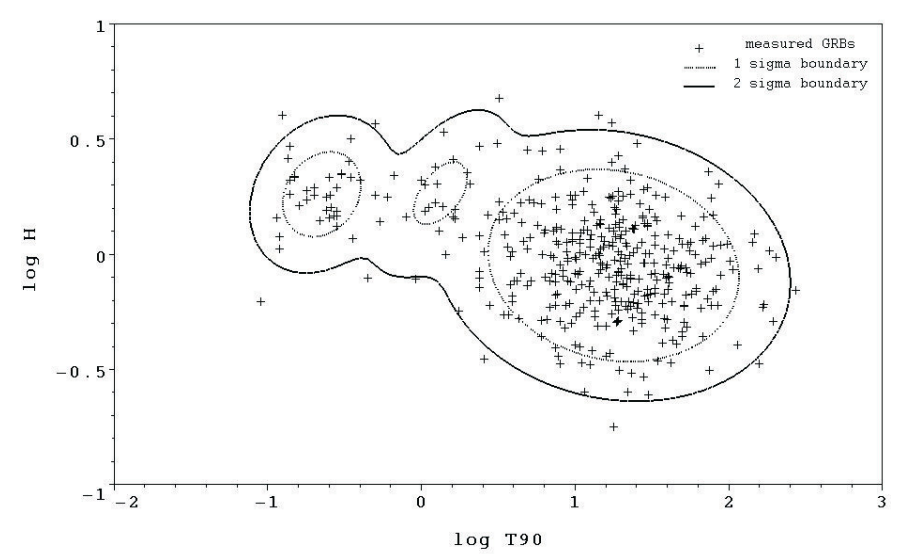

Fig. 4. Hardness ratio vs. $T_{90}$ of the RHESSI GRBs with the best fit of three bivariate log-normal functions.

introducing the third group being accidental, that are much lower than $5 \%$. Thus, the hypothesis of introducing the third group is highly acceptable.
Table 5. Parameters of the best fit with two and three bivariate lognormal functions done by the maximum likelihood method on the RHESSI data. $\mu_{x}$ are the means on the $x$-axis $\left(x=\log T_{90}\right), \mu_{y}$ are the means on the $y$-axis $(y=\log H), \sigma_{x}$ are the dispersions on the $x$-axis, $\sigma_{y}$ are the dispersions on the $y$-axis, $r$ are the correlation coefficients, $w$ are the weights of the distribution and $L_{2}, L_{3}$ are the likelihoods. Given uncertainties are standard deviations of the parameters obtained by ten different fittings of data sets, where the durations and hardness ratios were randomly changed by their uncertainties.

\begin{tabular}{lrr}
\hline \hline Parameter & 2 log-normal & 3 log-normal \\
\hline$\mu_{x, \text { short }}$ & $-0.38 \pm 0.018$ & $-0.65 \pm 0.018$ \\
$\mu_{y \text {,short }}$ & $0.26 \pm 0.010$ & $0.26 \pm 0.009$ \\
$\sigma_{x \text {, short }}$ & $0.42 \pm 0.012$ & $0.20 \pm 0.016$ \\
$\sigma_{y, \text { short }}$ & $0.15 \pm 0.009$ & $0.15 \pm 0.012$ \\
$w_{\text {short }}[\%]$ & $14.2 \pm 0.3$ & $9.2 \pm 0.5$ \\
$r_{\text {short }}$ & $0.14 \pm 0.092$ & $0.22 \pm 0.119$ \\
$\mu_{x, \text { long }}$ & $1.25 \pm 0.004$ & $1.25 \pm 0.006$ \\
$\mu_{y, \text { long }}$ & $-0.05 \pm 0.004$ & $-0.05 \pm 0.004$ \\
$\sigma_{x, \text { long }}$ & $0.42 \pm 0.004$ & $0.42 \pm 0.005$ \\
$\sigma_{y, \text { long }}$ & $0.22 \pm 0.003$ & $0.22 \pm 0.003$ \\
$w_{\text {long }}[\%]$ & $85.8 \pm 0.3$ & $85.5 \pm 0.8$ \\
$r_{\text {long }}$ & $-0.14 \pm 0.018$ & $-0.13 \pm 0.020$ \\
$\mu_{x \text {,middle }}$ & & $0.11 \pm 0.029$ \\
$\mu_{y, \text { middle }}$ & & $0.27 \pm 0.019$ \\
$\sigma_{x, \text { middle }}$ & & $0.21 \pm 0.057$ \\
$\sigma_{y, \text { middle }}$ & & $0.17 \pm 0.035$ \\
$w_{\text {middle }}[\%]$ & & $5.3 \pm 1.1$ \\
$r_{\text {middle }}$ & & $0.59 \pm 0.230$ \\
$\ln L_{2}$ & -323.91 & \\
$\ln L_{3}$ & & -313.00 \\
\hline
\end{tabular}

Table 6. The maximal likelihoods and corresponding probabilities that introducing of the third group is accidental for maximum likelihood fittings (two-dimensional) with two and three bivariate log-normal functions of ten different changes of durations and hardness ratios by their uncertainties.

\begin{tabular}{ccc}
\hline \hline $\ln L_{2}$ & $\ln L_{3}$ & $\begin{array}{c}\text { Probability } \\
{[\%]}\end{array}$ \\
\hline-349.40 & -339.92 & 0.42 \\
-348.28 & -336.36 & 0.06 \\
-350.66 & -340.80 & 0.31 \\
-347.12 & -338.15 & 0.64 \\
-349.64 & -340.47 & 0.54 \\
-344.59 & -338.17 & 4.56 \\
-350.36 & -342.47 & 1.50 \\
-344.44 & -334.71 & 0.35 \\
-349.37 & -336.51 & 0.03 \\
-348.06 & -338.57 & 0.42 \\
\hline
\end{tabular}

\section{Discussion}

The analysis of the one-dimensional duration distribution, by $\chi^{2}$ fitting, has revealed the class of so-called long GRBs (about $83 \%$ of all RHESSI GRBs) with typical durations from 5 to $70 \mathrm{~s}$, the most probable duration being $T_{90} \approx 19 \mathrm{~s}$. Another class are short GRBs (about $9 \%$ of all RHESSI GRBs) with typical durations from 0.1 to $0.4 \mathrm{~s}$, the most probable duration being $T_{90} \approx 0.21 \mathrm{~s}$. By fitting 3 log-normal functions, we have identified a third class (about $8 \%$ of all RHESSI GRBs) with typical durations from 0.8 to $3 \mathrm{~s}$, the most probable duration being $T_{90} \approx 1.5 \mathrm{~s}$. The 
Table 7. The RHESSI GRB data set including I. GRB names which correspond to dates (the letters after GRB names are internal and do not have to be in accordance with e.g. GCN GRB names), II. GRB peak time, III. $T_{90}$ duration, IV. time resolution $\delta t_{\text {res }}$ (described above) and V. hardness ratios.

\begin{tabular}{|c|c|c|c|c|}
\hline GRB & $\begin{array}{r}\text { Peak time } \\
\text { UTC } \\
\end{array}$ & $\begin{array}{r}T_{90} \\
{[\mathrm{~s}]} \\
\end{array}$ & $\begin{array}{r}\delta t_{\text {res }} \\
{[\mathrm{s}]} \\
\end{array}$ & $\begin{array}{r}\text { Hardness ratio } \\
\log H \\
\end{array}$ \\
\hline 020214 & $18: 49: 47.700$ & $E+1$ & $2.0 \mathrm{E}-1$ & E-1 \\
\hline & $19: 49: 41.750$ & $.40 \pm 0.06) \mathrm{E}+1$ & $5.0 \mathrm{E}-1$ & $0.11) \mathrm{E}-1$ \\
\hline 020302 & $12: 23: 54.400$ & $8 \pm 0.32) \mathrm{E}+1$ & $8.0 \mathrm{E}-1$ & $0.53) \mathrm{E}-1$ \\
\hline 020306 & $18: 58: 02.893$ & $(1.35 \pm 0.16) \mathrm{E}-1$ & $1.5 \mathrm{E}-2$ & $(4.16 \pm 0.40) \mathrm{E}-1$ \\
\hline 020311 & $01: 21: 31.550$ & & $3.0 \mathrm{E}-1$ & \\
\hline 020313 & 01:17:53.400 & & $4.0 \mathrm{E}-1$ & $-(5.17 \pm 0.50) \mathrm{E}-1$ \\
\hline 020315 & $15: 42: 47.550$ & $26 \pm 0.11) \mathrm{E}+1$ & $3.0 \mathrm{E}-1$ & $(2.97 \pm 6.94) \mathrm{E}-2$ \\
\hline & .750 & & $5.0 \mathrm{E}-1$ & 5) $\mathrm{E}-1$ \\
\hline 020407 & 04:14:44.300 & & $6.0 \mathrm{E}-1$ & $-(1.72 \pm 2.76) \mathrm{E}-2$ \\
\hline 020409 & $09: 27: 23.500$ & & $1.0 \mathrm{E}+0$ & 37) $\mathrm{E}-2$ \\
\hline & 500 & $\mathrm{E}+0$ & $2.0 \mathrm{E}-1$ & 57) $\mathrm{E}-2$ \\
\hline 020417 & $05: 36: 26.250$ & $\pm 0.52) \mathrm{E}+1$ & $5.0 \mathrm{E}-1$ & $-(1.62 \pm 0.58) \mathrm{E}-1$ \\
\hline & 8.850 & 3) $\mathrm{E}+0$ & $1.0 \mathrm{E}-1$ & 20)E-1 \\
\hline & & & $3.0 \mathrm{E}-2$ & \\
\hline & .650 & $(1$ & $3.0 \mathrm{E}-1$ & 2) $\mathrm{E}-1$ \\
\hline & 675 & & & 2) $\mathrm{E}-2$ \\
\hline 0 & & & $E-1$ & \\
\hline & & & $E-1$ & 5) $\mathrm{E}-1$ \\
\hline & & & $2.0 \mathrm{E}-2$ & \\
\hline 020 & 525 & & $5.0 \mathrm{E}-2$ & E-1 \\
\hline & 085 & & 7.0E-2 & 1)E-1 \\
\hline & & & $3.0 \mathrm{E}-2$ & \\
\hline & $14: 1$ & & $3.0 \mathrm{E}-1$ & 9)E-2 \\
\hline & 875 & & $\mathrm{E}-2$ & 6) $\mathrm{E}-1$ \\
\hline & & & $1.0 \mathrm{E}-1$ & E-1 \\
\hline & 650 & & $3.0 \mathrm{E}-1$ & 6) $\mathrm{E}-1$ \\
\hline & & & E-1 & \\
\hline & & & $1.0 \mathrm{E}+0$ & $-(3$ \\
\hline & & & & \\
\hline & & & -2 & E-1 \\
\hline 020 & 100 & & $2.0 \mathrm{E}-1$ & E-1 \\
\hline & & & & \\
\hline & & & $1.0 \mathrm{E}-1$ & E-2 \\
\hline 020819A & 455 & & $3.0 \mathrm{E}-2$ & ) $\mathrm{E}-1$ \\
\hline & & & & E-1 1 \\
\hline & & & & \\
\hline & & & $3.0 \mathrm{E}-1$ & E-1 \\
\hline & & & -1 & E-1 \\
\hline & & & $8.0 \mathrm{E}-1$ & E-1 \\
\hline & & & & \\
\hline & & & & \\
\hline & 250 & $E+1$ & $5.0 \mathrm{E}-1$ & $-(2.94=$ \\
\hline & & & & \\
\hline & & & $3.0 \mathrm{E}-1$ & 6) $\mathrm{E}-2$ \\
\hline & 7.300 & +1 & $2.0 \mathrm{E}-1$ & 5) $\mathrm{E}-2$ \\
\hline & & & & \\
\hline & & & $E-1$ & E-1 \\
\hline & & & $1.0 \mathrm{E}-1$ & 9) $\mathrm{E}-2$ \\
\hline & & & & \\
\hline & 9.000 & +1 & $4.0 \mathrm{E}-1$ & $\pm 5.23) \mathrm{E}-2$ \\
\hline & & & & \\
\hline & & & $3.0 \mathrm{E}-1$ & 1)E-1 \\
\hline & $12: 5$ & +1 & $2.0 \mathrm{E}-1$ & $-(1.8)$ \\
\hline & & & & \\
\hline & & & $10 F_{2}$ & \\
\hline & $18: 29.750$ & $(7.65 \pm 0.33) \mathrm{E}+1$ & $1.5 \mathrm{E}+0$ & $-(2.23 \pm 0.36) \mathrm{E}-1$ \\
\hline & & & & \\
\hline & & & $8.0 \mathrm{E}-2$ & 8) $\mathrm{E}-1$ \\
\hline 021214 & $03: 27: 25.500$ & $(3.00 \pm 0.28) \mathrm{E}+1$ & $1.0 \mathrm{E}+0$ & $-(2.07 \pm 0.77) \mathrm{E}-1$ \\
\hline & & & $1.0 \mathrm{E}-1$ & $-(2.92 \pm 0.77) \mathrm{E}-1$ \\
\hline & & & $5.0 \mathrm{E}-2$ & .43)E-1 \\
\hline 030102 & $23: 18: 59.350$ & $(1.32 \pm 0.09) \mathrm{E}+1$ & $3.0 \mathrm{E}-1$ & $-(1.65 \pm 0.56) \mathrm{E}-1$ \\
\hline
\end{tabular}

existence of the intermediate class from the RHESSI $T_{90}$ distribution is not confirmed to a sufficiently high significance using only the $\chi^{2}$ fit. However, the maximum likelihood ratio test on
Table 7. continued.

\begin{tabular}{|c|c|c|c|c|}
\hline GRB & $\begin{array}{r}\text { Peak time } \\
\text { UTC }\end{array}$ & $\begin{array}{r}T_{90} \\
{[\mathrm{~s}]}\end{array}$ & $\begin{array}{r}\delta t_{\text {res }} \\
{[\mathrm{s}]}\end{array}$ & $\begin{array}{r}\text { Hardness ratio } \\
\log H\end{array}$ \\
\hline 030103 & $21: 46: 46.750$ & $(1.00 \pm 0.12) \mathrm{E}+1$ & $5.0 \mathrm{E}-1$ & $-(3.93 \pm 1.14) \mathrm{E}-1$ \\
\hline 030105 & $14: 34: 11.995$ & $(1.23 \pm 0.06) \mathrm{E}+0$ & $3.0 \mathrm{E}-2$ & $(3.77 \pm 0.40) \mathrm{E}-1$ \\
\hline 030110 & $09: 39: 30.205$ & $(9.00 \pm 3.14) \mathrm{E}-2$ & $3.0 \mathrm{E}-2$ & $-(2.04 \pm 0.88) \mathrm{E}-1$ \\
\hline 030115A & $06: 25: 37.250$ & $(7.95 \pm 0.14) \mathrm{E}+1$ & $5.0 \mathrm{E}-1$ & $(3.54 \pm 1.40) \mathrm{E}-2$ \\
\hline 030115B & $08: 15: 48.500$ & $(2.70 \pm 0.10) \mathrm{E}+1$ & $2.0 \mathrm{E}-1$ & $-(2.83 \pm 0.36) \mathrm{E}-1$ \\
\hline 030127 & $12: 32: 42.750$ & $(3.80 \pm 0.40) \mathrm{E}+1$ & $5.0 \mathrm{E}-1$ & $(2.34 \pm 8.30) \mathrm{E}-2$ \\
\hline 030204 & $12: 45: 36.500$ & $(5.60 \pm 0.13) \mathrm{E}+1$ & $1.0 \mathrm{E}+0$ & $(1.39 \pm 1.34) \mathrm{E}-2$ \\
\hline 030206 & 11:00:32.010 & $(1.40 \pm 0.24) \mathrm{E}-1$ & $2.0 \mathrm{E}-2$ & $(2.62 \pm 0.83) \mathrm{E}-1$ \\
\hline 030212 & $22: 17: 18.450$ & $(2.31 \pm 0.26) \mathrm{E}+1$ & $3.0 \mathrm{E}-1$ & $-(2.25 \pm 0.97) \mathrm{E}-1$ \\
\hline 030214 & $14: 48: 21.325$ & $(1.83 \pm 0.10) \mathrm{E}+1$ & $1.5 \mathrm{E}-1$ & $-(2.07 \pm 0.49) \mathrm{E}-1$ \\
\hline 030216 & $16: 13: 44.150$ & $(9.30 \pm 1.40) \mathrm{E}+0$ & $3.0 \mathrm{E}-1$ & $-(1.20 \pm 1.16) \mathrm{E}-1$ \\
\hline 030217 & $02: 45: 42.600$ & $(5.40 \pm 0.08) \mathrm{E}+1$ & $8.0 \mathrm{E}-2$ & $(4.46 \pm 1.28) \mathrm{E}-2$ \\
\hline 030222 & $16: 32: 53.250$ & $(1.44 \pm 0.18) \mathrm{E}+1$ & $3.0 \mathrm{E}-1$ & $-(2.01 \pm 1.02) \mathrm{E}-1$ \\
\hline 030223 & $09: 45: 15.250$ & $(2.05 \pm 0.11) \mathrm{E}+1$ & $5.0 \mathrm{E}-1$ & $(2.50 \pm 0.42) \mathrm{E}-1$ \\
\hline 030225 & $15: 02: 53.750$ & $(2.00 \pm 0.11) \mathrm{E}+1$ & $5.0 \mathrm{E}-1$ & $-(9.23 \pm 4.20) \mathrm{E}-2$ \\
\hline 030227 & $13: 12: 05.250$ & $(7.10 \pm 1.03) \mathrm{E}+1$ & $5.0 \mathrm{E}-1$ & $(0.68 \pm 1.11) \mathrm{E}-1$ \\
\hline 030228 & $20: 26: 47.250$ & $(2.80 \pm 0.18) \mathrm{E}+1$ & $5.0 \mathrm{E}-1$ & $-(3.96 \pm 4.98) \mathrm{E}-2$ \\
\hline 030301 & $20: 27: 21.500$ & $(4.30 \pm 0.35) \mathrm{E}+1$ & $1.0 \mathrm{E}+0$ & $-(3.73 \pm 0.85) \mathrm{E}-1$ \\
\hline 030306 & $03: 38: 16.150$ & $(1.26 \pm 0.04) \mathrm{E}+1$ & $3.0 \mathrm{E}-1$ & $-(1.16 \pm 0.22) \mathrm{E}-1$ \\
\hline 030307 & $14: 31: 58.950$ & $(3.80 \pm 0.11) \mathrm{E}+0$ & $1.0 \mathrm{E}-1$ & $-(1.34 \pm 0.13) \mathrm{E}-1$ \\
\hline 030320A & $10: 12: 01.150$ & $(4.86 \pm 0.15) \mathrm{E}+1$ & $3.0 \mathrm{E}-1$ & $-(6.10 \pm 2.51) \mathrm{E}-2$ \\
\hline 030320B & $18: 49: 35.500$ & $(1.56 \pm 0.06) \mathrm{E}+2$ & $3.0 \mathrm{E}+0$ & $-(6.38 \pm 2.83) \mathrm{E}-2$ \\
\hline 030326 & $10: 43: 44.150$ & $(1.26 \pm 0.06) \mathrm{E}+1$ & $3.0 \mathrm{E}-1$ & $-(2.01 \pm 0.34) \mathrm{E}-1$ \\
\hline 030328 & $07: 28: 48.250$ & $(6.55 \pm 0.26) \mathrm{E}+1$ & $5.0 \mathrm{E}-1$ & $-(4.09 \pm 3.25) \mathrm{E}-2$ \\
\hline 030329A & $11: 37: 40.850$ & $(1.74 \pm 0.03) \mathrm{E}+1$ & $3.0 \mathrm{E}-1$ & $-(2.60 \pm 0.06) \mathrm{E}-1$ \\
\hline 030329B & $15: 34: 18.500$ & $(6.10 \pm 0.29) \mathrm{E}+1$ & $1.0 \mathrm{E}+0$ & $-(1.50 \pm 0.39) \mathrm{E}-1$ \\
\hline 030331 & $05: 38: 49.500$ & $(2.38 \pm 0.11) \mathrm{E}+1$ & $2.0 \mathrm{E}-1$ & $-(3.34 \pm 0.47) \mathrm{E}-1$ \\
\hline 030406 & $22: 42: 57.450$ & $(7.02 \pm 0.13) \mathrm{E}+1$ & $3.0 \mathrm{E}-1$ & $-(3.30 \pm 1.54) \mathrm{E}-2$ \\
\hline 030410 & $11: 23: 42.275$ & $(1.60 \pm 0.16) \mathrm{E}+0$ & $5.0 \mathrm{E}-2$ & $(4.10 \pm 1.01) \mathrm{E}-1$ \\
\hline 030413 & $07: 34: 45.550$ & $(2.04 \pm 0.09) \mathrm{E}+1$ & 3.0E-1 & $(1.48 \pm 0.35) \mathrm{E}-1$ \\
\hline 030414 & $13: 48: 28.250$ & $(2.85 \pm 0.08) \mathrm{E}+1$ & $5.0 \mathrm{E}-1$ & $-(3.66 \pm 1.80) \mathrm{E}-2$ \\
\hline 030419 & $01: 12: 14.300$ & $(3.78 \pm 0.06) \mathrm{E}+1$ & $2.0 \mathrm{E}-1$ & $-(2.81 \pm 0.14) \mathrm{E}-1$ \\
\hline 030421 & $00: 36: 32.350$ & $(1.27 \pm 0.04) \mathrm{E}+1$ & $1.0 \mathrm{E}-1$ & $(8.23 \pm 2.40) \mathrm{E}-2$ \\
\hline 030422 & 09:01:30.850 & $(1.47 \pm 0.11) \mathrm{E}+1$ & $3.0 \mathrm{E}-1$ & $-(3.83 \pm 6.08) \mathrm{E}-2$ \\
\hline 030428 & $22: 31: 23.300$ & $(9.60 \pm 0.26) \mathrm{E}+0$ & $2.0 \mathrm{E}-1$ & $(5.70 \pm 1.42) \mathrm{E}-2$ \\
\hline 030501A & $01: 17: 22.300$ & $(7.40 \pm 0.49) \mathrm{E}+0$ & $2.0 \mathrm{E}-1$ & $-(2.21 \pm 0.55) \mathrm{E}-1$ \\
\hline 030501B & $03: 10: 23.850$ & $(1.17 \pm 0.16) \mathrm{E}+1$ & 3.0E-1 & $-(4.00 \pm 1.43) \mathrm{E}-1$ \\
\hline 030501C & $20: 44: 49.520$ & $(1.24 \pm 0.12) \mathrm{E}+0$ & 4.0E-2 & $(2.21 \pm 0.74) \mathrm{E}-1$ \\
\hline $030505 \mathrm{~A}$ & $07: 39: 13.100$ & $(8.82 \pm 0.43) \mathrm{E}+1$ & $6.0 \mathrm{E}-1$ & $(3.77 \pm 3.96) \mathrm{E}-2$ \\
\hline 030505B & $09: 03: 26.750$ & $(1.07 \pm 0.06) \mathrm{E}+2$ & $1.5 \mathrm{E}+0$ & $-(6.53 \pm 4.77) \mathrm{E}-2$ \\
\hline 030506 & $02: 04: 27.350$ & $(2.31 \pm 0.07) \mathrm{E}+1$ & $3.0 \mathrm{E}-1$ & $-(9.31 \pm 2.34) \mathrm{E}-2$ \\
\hline 030518A & $01: 23: 49.650$ & $(2.52 \pm 0.02) \mathrm{E}+1$ & $1.0 \mathrm{E}-1$ & $(4.79 \pm 0.07) \mathrm{E}-1$ \\
\hline 030518B & $03: 12: 23.050$ & $(1.74 \pm 0.12) \mathrm{E}+1$ & $3.0 \mathrm{E}-1$ & $(3.98 \pm 0.54) \mathrm{E}-1$ \\
\hline 030519A & $09: 32: 22.700$ & $(3.20 \pm 0.39) \mathrm{E}+0$ & $2.0 \mathrm{E}-1$ & $(6.78 \pm 1.13) \mathrm{E}-1$ \\
\hline 030519B & 14:05:00.100 & $(9.00 \pm 0.21) \mathrm{E}+0$ & $2.0 \mathrm{E}-1$ & $-(1.81 \pm 0.47) \mathrm{E}-2$ \\
\hline 030523 & 14:10:53.190 & $(1.20 \pm 0.26) \mathrm{E}-1$ & $2.0 \mathrm{E}-2$ & $(0.22 \pm 1.04) \mathrm{E}-1$ \\
\hline 030528 & 13:03:07.750 & $(2.15 \pm 0.17) \mathrm{E}+1$ & $5.0 \mathrm{E}-1$ & $-(2.26 \pm 0.69) \mathrm{E}-1$ \\
\hline 030601 & $22: 12: 06.050$ & $(1.83 \pm 0.08) \mathrm{E}+1$ & $3.0 \mathrm{E}-1$ & $(7.59 \pm 3.59) \mathrm{E}-2$ \\
\hline 030614 & $01: 30: 42.500$ & $(1.68 \pm 0.05) \mathrm{E}+2$ & $3.0 \mathrm{E}+0$ & $-(2.19 \pm 0.21) \mathrm{E}-1$ \\
\hline 030626 & $01: 46: 54.950$ & $(3.85 \pm 0.10) \mathrm{E}+1$ & $7.0 \mathrm{E}-1$ & $(2.46 \pm 0.18) \mathrm{E}-1$ \\
\hline 030703 & $19: 14: 02.400$ & $(5.68 \pm 0.74) \mathrm{E}+1$ & $8.0 \mathrm{E}-1$ & $-(0.84 \pm 1.03) \mathrm{E}-1$ \\
\hline 030706 & $00: 02: 17.750$ & $(4.50 \pm 0.45) \mathrm{E}+0$ & 3.0E-1 & $(1.36 \pm 0.62) \mathrm{E}-1$ \\
\hline 030710 & 23:05:02.350 & $(1.77 \pm 0.07) \mathrm{E}+1$ & $3.0 \mathrm{E}-1$ & $-(7.49 \pm 0.71) \mathrm{E}-1$ \\
\hline 030714 & $22: 14: 50.300$ & $(1.02 \pm 0.08) \mathrm{E}+1$ & $2.0 \mathrm{E}-1$ & $-(2.73 \pm 0.73) \mathrm{E}-1$ \\
\hline 030716 & 11:57:19.750 & $(5.35 \pm 0.40) \mathrm{E}+1$ & $5.0 \mathrm{E}-1$ & $-(3.16 \pm 0.75) \mathrm{E}-1$ \\
\hline 030721 & $23: 41: 10.100$ & $(2.96 \pm 0.05) \mathrm{E}+1$ & $2.0 \mathrm{E}-1$ & $(2.88 \pm 0.14) \mathrm{E}-1$ \\
\hline 030725 & $11: 46: 27.800$ & $(1.84 \pm 0.07) \mathrm{E}+1$ & $4.0 \mathrm{E}-1$ & $-(2.22 \pm 0.32) \mathrm{E}-1$ \\
\hline 030726A & $06: 38: 41.750$ & $(2.75 \pm 0.06) \mathrm{E}+1$ & $5.0 \mathrm{E}-1$ & $(1.56 \pm 0.11) \mathrm{E}-1$ \\
\hline 030726B & $09: 51: 37.500$ & $(1.94 \pm 0.12) \mathrm{E}+2$ & $1.0 \mathrm{E}+0$ & $-(2.91 \pm 0.63) \mathrm{E}-1$ \\
\hline 030728 & 09:05:55.100 & $(1.92 \pm 0.28) \mathrm{E}+1$ & $2.0 \mathrm{E}-1$ & $(4.26 \pm 1.50) \mathrm{E}-1$ \\
\hline 030824 & $06: 31: 10.850$ & $(3.00 \pm 0.20) \mathrm{E}+1$ & $3.0 \mathrm{E}-1$ & $-(6.11 \pm 0.95) \mathrm{E}-1$ \\
\hline 030827 & $16: 08: 40.325$ & $(1.85 \pm 0.08) \mathrm{E}+0$ & $5.0 \mathrm{E}-2$ & $(7.30 \pm 2.77) \mathrm{E}-2$ \\
\hline 030830 & $18: 37: 46.250$ & $(2.05 \pm 0.09) \mathrm{E}+1$ & $5.0 \mathrm{E}-1$ & $-(1.27 \pm 0.34) \mathrm{E}-1$ \\
\hline 030831 & $15: 07: 22.450$ & $(2.31 \pm 0.09) \mathrm{E}+1$ & $3.0 \mathrm{E}-1$ & $-(1.04 \pm 0.33) \mathrm{E}-1$ \\
\hline 030919 & $21: 10: 36.750$ & $(2.00 \pm 0.14) \mathrm{E}+1$ & $5.0 \mathrm{E}-1$ & $-(2.05 \pm 0.59) \mathrm{E}-1$ \\
\hline 030921 & $08: 38: 23.650$ & $(1.47 \pm 0.06) \mathrm{E}+1$ & $3.0 \mathrm{E}-1$ & $(2.23 \pm 3.15) \mathrm{E}-2$ \\
\hline
\end{tabular}

the same data reveals that the introduction of a third class is statistically significant. The $\chi^{2}$ method might not be as sensitive and hence robust as the likelihood method, because of the low 
Table 7. continued.

\begin{tabular}{|c|c|c|c|c|}
\hline GRB & $\begin{array}{r}\text { Peak time } \\
\text { UTC }\end{array}$ & $\begin{array}{r}T_{90} \\
{[\mathrm{~s}]}\end{array}$ & $\begin{array}{r}\delta t_{\text {res }} \\
{[\mathrm{s}]}\end{array}$ & $\begin{array}{r}\text { Hardness ratio } \\
\qquad \log H\end{array}$ \\
\hline 030922A & $08: 43: 35.950$ & $(2.28 \pm 0.08) \mathrm{E}+1$ & $3.0 \mathrm{E}-1$ & $-(1.07 \pm 0.30) \mathrm{E}-1$ \\
\hline 030922B & $18: 30: 56.900$ & $(1.08 \pm 0.03) \mathrm{E}+1$ & $2.0 \mathrm{E}-1$ & $(4.63 \pm 1.41) \mathrm{E}-2$ \\
\hline 030926 & $16: 52: 28.290$ & $(2.80 \pm 0.31) \mathrm{E}-1$ & $2.0 \mathrm{E}-2$ & $(2.89 \pm 0.77) \mathrm{E}-1$ \\
\hline 031005 & $08: 21: 50.250$ & $(1.60 \pm 0.18) \mathrm{E}+1$ & $5.0 \mathrm{E}-1$ & $-(4.44 \pm 1.19) \mathrm{E}-1$ \\
\hline 031019 & $22: 00: 57.175$ & $(7.50 \pm 0.71) \mathrm{E}+0$ & $1.5 \mathrm{E}-1$ & $(2.48 \pm 0.79) \mathrm{E}-1$ \\
\hline 031024 & $09: 24: 14.350$ & $(4.00 \pm 0.32) \mathrm{E}+0$ & $1.0 \mathrm{E}-1$ & $(1.77 \pm 0.58) \mathrm{E}-1$ \\
\hline 031027 & $17: 07: 50.250$ & $(3.90 \pm 0.07) \mathrm{E}+1$ & $5.0 \mathrm{E}-1$ & $-(1.03 \pm 0.10) \mathrm{E}-1$ \\
\hline 031107 & $18: 24: 07.400$ & $(1.92 \pm 0.06) \mathrm{E}+1$ & $4.0 \mathrm{E}-1$ & $(1.02 \pm 2.13) \mathrm{E}-2$ \\
\hline 031108 & $14: 11: 19.250$ & $(2.75 \pm 0.07) E+1$ & $5.0 \mathrm{E}-1$ & $(1.67 \pm 0.16) \mathrm{E}-1$ \\
\hline 031111 & $16: 45: 20.920$ & $(3.20 \pm 0.08) \mathrm{E}+0$ & $8.0 \mathrm{E}-2$ & $(1.50 \pm 0.08) \mathrm{E}-1$ \\
\hline 031118 & $06: 26: 16.410$ & $(2.60 \pm 0.27) \mathrm{E}-1$ & $2.0 \mathrm{E}-2$ & $(2.01 \pm 0.57) \mathrm{E}-1$ \\
\hline 031120 & $05: 52: 38.250$ & $(9.75 \pm 0.30) \mathrm{E}+1$ & $1.5 \mathrm{E}+0$ & $(4.24 \pm 2.27) \mathrm{E}-2$ \\
\hline 031127 & $18: 58: 55.050$ & $(1.92 \pm 0.17) \mathrm{E}+1$ & $3.0 \mathrm{E}-1$ & $-(2.88 \pm 0.83) \mathrm{E}-1$ \\
\hline 031130 & 02:04:53.775 & $(4.65 \pm 0.29) \mathrm{E}+0$ & $1.5 \mathrm{E}-1$ & $(5.52 \pm 4.44) \mathrm{E}-2$ \\
\hline 031214 & $22: 50: 44.150$ & $(8.30 \pm 1.01) \mathrm{E}+0$ & $1.0 \mathrm{E}-1$ & $-(6.98 \pm 9.55) \mathrm{E}-2$ \\
\hline 031218 & $06: 28: 09.370$ & $(2.20 \pm 0.24) \mathrm{E}-1$ & $2.0 \mathrm{E}-2$ & $(1.46 \pm 0.51) \mathrm{E}-1$ \\
\hline 031219 & 05:39:05.850 & $(7.50 \pm 0.21) \mathrm{E}+0$ & $1.0 \mathrm{E}-1$ & $-(1.21 \pm 0.21) \mathrm{E}-1$ \\
\hline 031226A & $15: 43: 08.750$ & $(3.45 \pm 0.29) \mathrm{E}+1$ & $5.0 \mathrm{E}-1$ & $-(4.63 \pm 1.03) \mathrm{E}-1$ \\
\hline 031226B & $17: 51: 29.750$ & $(4.40 \pm 0.23) \mathrm{E}+1$ & $5.0 \mathrm{E}-1$ & $-(5.74 \pm 4.22) \mathrm{E}-2$ \\
\hline 040102 & $19: 35: 26.975$ & $(7.80 \pm 0.83) \mathrm{E}+0$ & $1.5 \mathrm{E}-1$ & $-(4.43 \pm 1.15) \mathrm{E}-1$ \\
\hline 040108 & $07: 46: 39.900$ & $(3.30 \pm 0.37) \mathrm{E}+1$ & $6.0 \mathrm{E}-1$ & $-(2.64 \pm 1.03) \mathrm{E}-1$ \\
\hline 040113 & $01: 36: 46.350$ & $(1.62 \pm 0.10) E+1$ & $3.0 \mathrm{E}-1$ & $-(4.60 \pm 5.01) \mathrm{E}-2$ \\
\hline 040115 & 18:30:11.550 & $(2.19 \pm 0.24) \mathrm{E}+1$ & $3.0 \mathrm{E}-1$ & $-(2.40 \pm 0.94) \mathrm{E}-1$ \\
\hline 040125 & $22: 14: 47.100$ & $(1.60 \pm 0.23) \mathrm{E}+1$ & $2.0 \mathrm{E}-1$ & $-(1.67 \pm 1.16) \mathrm{E}-1$ \\
\hline 040205A & $05: 19: 47.350$ & $(3.90 \pm 0.62) \mathrm{E}+0$ & $1.0 \mathrm{E}-1$ & $-(2.06 \pm 1.29) \mathrm{E}-1$ \\
\hline 040205B & $09: 27: 45.750$ & $(2.65 \pm 0.33) \mathrm{E}+1$ & $5.0 \mathrm{E}-1$ & $(2.19 \pm 0.98) \mathrm{E}-1$ \\
\hline 040207 & $22: 12: 22.600$ & $(2.44 \pm 0.06) \mathrm{E}+1$ & $4.0 \mathrm{E}-1$ & $(1.07 \pm 0.17) \mathrm{E}-1$ \\
\hline 040211 & $15: 02: 08.050$ & $(3.70 \pm 0.52) \mathrm{E}+0$ & $1.0 \mathrm{E}-1$ & $-(2.61 \pm 1.25) \mathrm{E}-1$ \\
\hline 040215 & $00: 28: 02.500$ & $(5.00 \pm 0.29) \mathrm{E}+1$ & $1.0 \mathrm{E}+0$ & $-(3.57 \pm 0.57) \mathrm{E}-1$ \\
\hline & $00: 55: 15.800$ & $(1.72 \pm 0.09) \mathrm{E}+1$ & $4.0 \mathrm{E}-1$ & $(5.71 \pm 0.46) \mathrm{E}-1$ \\
\hline $040225 \mathrm{~A}$ & $05: 30: 53.850$ & $(1.05 \pm 0.11) \mathrm{E}+1$ & $3.0 \mathrm{E}-1$ & $-(8.84 \pm 8.12) \mathrm{E}-2$ \\
\hline 040225B & 10:02:12.200 & $(1.40 \pm 0.16) \mathrm{E}+1$ & $4.0 \mathrm{E}-1$ & $-(3.12 \pm 1.10) \mathrm{E}-1$ \\
\hline 040228 & 00:09:08.500 & $(2.73 \pm 0.02) \mathrm{E}+2$ & $2.0 \mathrm{E}-1$ & $-(1.58 \pm 0.07) \mathrm{E}-1$ \\
\hline 040302A & $04: 14: 35.250$ & $(2.37 \pm 0.27) \mathrm{E}+1$ & $3.0 \mathrm{E}-1$ & $-(2.76 \pm 1.05) \mathrm{E}-1$ \\
\hline 040302B & $12: 24: 03.900$ & $(1.08 \pm 0.02) \mathrm{E}+1$ & $2.0 \mathrm{E}-1$ & $-(7.71 \pm 0.64) \mathrm{E}-2$ \\
\hline 040303 & $15: 32: 37.750$ & $(2.97 \pm 0.27) \mathrm{E}+1$ & $3.0 \mathrm{E}-1$ & $-(1.52 \pm 0.76) \mathrm{E}-1$ \\
\hline 040312 & $00: 02: 36.550$ & $(1.60 \pm 0.22) \mathrm{E}-1$ & $2.0 \mathrm{E}-2$ & $(2.13 \pm 0.48) \mathrm{E}-1$ \\
\hline 040316 & $18: 16: 14.350$ & $(1.50 \pm 0.02) \mathrm{E}+1$ & $1.0 \mathrm{E}-1$ & $-(4.87 \pm 1.30) \mathrm{E}-2$ \\
\hline 040323 & 13:03:04.750 & $(1.89 \pm 0.19) \mathrm{E}+1$ & $3.0 \mathrm{E}-1$ & $-(2.92 \pm 0.94) \mathrm{E}-1$ \\
\hline 040324 & $10: 21: 12.908$ & $(2.55 \pm 0.17) \mathrm{E}-1$ & $1.5 \mathrm{E}-2$ & $(2.54 \pm 0.26) \mathrm{E}-1$ \\
\hline 040327 & $16: 19: 27.500$ & $(2.00 \pm 0.32) \mathrm{E}+1$ & $1.0 \mathrm{E}+0$ & $-(4.06 \pm 1.54) \mathrm{E}-1$ \\
\hline 040329 & 11:10:51.965 & $(2.07 \pm 0.04) \mathrm{E}+0$ & $3.0 \mathrm{E}-2$ & $(3.06 \pm 0.12) \mathrm{E}-1$ \\
\hline 040330 & $13: 14: 42.150$ & $(2.88 \pm 0.41) \mathrm{E}+1$ & $3.0 \mathrm{E}-1$ & $(0.04 \pm 1.09) \mathrm{E}-1$ \\
\hline 040404 & $10: 58: 52.150$ & $(4.90 \pm 0.35) \mathrm{E}+0$ & $1.0 \mathrm{E}-1$ & $-(1.38 \pm 0.60) \mathrm{E}-1$ \\
\hline 040413 & 13:09:56.490 & $(2.80 \pm 0.31) \mathrm{E}-1$ & $2.0 \mathrm{E}-2$ & $(1.21 \pm 0.68) \mathrm{E}-1$ \\
\hline 040414 & 11:09:22.500 & $(7.70 \pm 0.24) \mathrm{E}+1$ & $1.0 \mathrm{E}+0$ & $-(1.63 \pm 2.36) \mathrm{E}-2$ \\
\hline 040421 & $02: 30: 27.300$ & $(1.14 \pm 0.02) \mathrm{E}+1$ & $2.0 \mathrm{E}-1$ & $(2.73 \pm 0.08) \mathrm{E}-1$ \\
\hline 040423 & $02: 23: 30.350$ & $(4.20 \pm 0.53) \mathrm{E}+0$ & 3.0E-1 & $-(1.16 \pm 0.87) \mathrm{E}-1$ \\
\hline 040425 & $16: 23: 34.275$ & $(8.10 \pm 0.19) \mathrm{E}+0$ & $5.0 \mathrm{E}-2$ & $(1.08 \pm 0.20) \mathrm{E}-1$ \\
\hline 040427 & $20: 12: 37.700$ & $(8.00 \pm 0.77) \mathrm{E}+0$ & $2.0 \mathrm{E}-1$ & $-(4.55 \pm 7.43) \mathrm{E}-2$ \\
\hline 040429 & $10: 53: 04.400$ & $(2.52 \pm 0.19) \mathrm{E}+1$ & $4.0 \mathrm{E}-1$ & $(2.85 \pm 6.00) \mathrm{E}-2$ \\
\hline 040502A & 06:37:06.900 & $(1.86 \pm 0.04) \mathrm{E}+1$ & $2.0 \mathrm{E}-1$ & $-(2.97 \pm 0.22) \mathrm{E}-1$ \\
\hline 040502B & $13: 30: 02.500$ & $(1.66 \pm 0.07) \mathrm{E}+2$ & $1.0 \mathrm{E}+0$ & $-(2.32 \pm 0.39) \mathrm{E}-1$ \\
\hline 040506 & $23: 45: 18.800$ & $(6.56 \pm 0.42) \mathrm{E}+1$ & $8.0 \mathrm{E}-1$ & $-(2.34 \pm 0.58) \mathrm{E}-1$ \\
\hline 040508 & $10: 15: 44.750$ & $(4.05 \pm 0.56) \mathrm{E}+1$ & $5.0 \mathrm{E}-1$ & $-(0.95 \pm 1.08) \mathrm{E}-1$ \\
\hline 040510 & 09:59:37.300 & $(6.00 \pm 0.57) \mathrm{E}+0$ & $2.0 \mathrm{E}-1$ & $-(2.87 \pm 0.83) \mathrm{E}-1$ \\
\hline 040513 & 03:02:17.500 & $(5.30 \pm 0.83) \mathrm{E}+1$ & $1.0 \mathrm{E}+0$ & $-(0.78 \pm 1.20) \mathrm{E}-1$ \\
\hline 040526 & $20: 21: 13.350$ & $(1.29 \pm 0.19) \mathrm{E}+1$ & $3.0 \mathrm{E}-1$ & $-(4.19 \pm 1.53) \mathrm{E}-1$ \\
\hline 040528 & $16: 55: 58.150$ & $(2.16 \pm 0.07) \mathrm{E}+1$ & $3.0 \mathrm{E}-1$ & $-(1.06 \pm 0.26) \mathrm{E}-1$ \\
\hline 040531 & $23: 15: 04.750$ & $(4.20 \pm 0.33) \mathrm{E}+1$ & $5.0 \mathrm{E}-1$ & $-(4.73 \pm 0.91) \mathrm{E}-1$ \\
\hline 040601 & $06: 33: 24.625$ & $(2.73 \pm 0.29) \mathrm{E}+1$ & $2.5 \mathrm{E}-1$ & $(5.10 \pm 8.45) \mathrm{E}-2$ \\
\hline 040603A & $15: 40: 58.700$ & $(1.24 \pm 0.13) \mathrm{E}+1$ & $2.0 \mathrm{E}-1$ & $-(1.42 \pm 0.84) \mathrm{E}-1$ \\
\hline 040603B & $19: 15: 38.500$ & $(7.50 \pm 1.00) \mathrm{E}+1$ & $3.0 \mathrm{E}+0$ & $-(5.04 \pm 1.49) \mathrm{E}-1$ \\
\hline 040605A & $04: 31: 44.500$ & $(5.80 \pm 0.73) \mathrm{E}+0$ & $2.0 \mathrm{E}-1$ & $(3.25 \pm 1.04) \mathrm{E}-1$ \\
\hline 040605B & $18: 46: 16.390$ & $(1.80 \pm 0.22) \mathrm{E}-1$ & $2.0 \mathrm{E}-2$ & $(2.33 \pm 0.48) \mathrm{E}-1$ \\
\hline $040605 \mathrm{C}$ & $23: 58: 45.800$ & $(1.68 \pm 0.08) \mathrm{E}+1$ & $4.0 \mathrm{E}-1$ & $-(4.30 \pm 0.50) \mathrm{E}-1$ \\
\hline
\end{tabular}

number of bursts in our data-sample (Horváth et al. 2008, 2nd section, 1st paragraph).

The hardness ratio versus duration plot for the RHESSI sample demonstrates further the existence of a third class. The
Table 7. continued.

\begin{tabular}{|c|c|c|c|c|}
\hline GRB & $\begin{array}{r}\text { Peak time } \\
\text { UTC }\end{array}$ & $\begin{array}{r}T_{90} \\
{[\mathrm{~s}]}\end{array}$ & $\begin{array}{r}\delta t_{\text {res }} \\
{[\mathrm{s}]}\end{array}$ & $\begin{array}{r}\text { Hardness ratio } \\
\log H\end{array}$ \\
\hline 040611 & $13: 36: 00.600$ & $(1.80 \pm 0.10) \mathrm{E}+1$ & $4.0 \mathrm{E}-1$ & $(2.02 \pm 0.44) \mathrm{E}-1$ \\
\hline 040619 & $15: 15: 52.250$ & $(7.70 \pm 0.27) \mathrm{E}+0$ & $1.0 \mathrm{E}-1$ & $-(1.74 \pm 0.28) \mathrm{E}-1$ \\
\hline 040701 & $22: 46: 45.250$ & $(9.60 \pm 0.69) \mathrm{E}+0$ & $3.0 \mathrm{E}-1$ & $(9.16 \pm 5.34) \mathrm{E}-2$ \\
\hline 040719 & $01: 16: 31.250$ & $(7.50 \pm 0.65) \mathrm{E}+0$ & $1.0 \mathrm{E}-1$ & $-(1.77 \pm 0.73) \mathrm{E}-1$ \\
\hline 040723 & $04: 06: 38.150$ & $(1.02 \pm 0.04) \mathrm{E}+1$ & $1.0 \mathrm{E}-1$ & $-(1.51 \pm 0.27) \mathrm{E}-1$ \\
\hline 040731 & $10: 24: 42.750$ & $(2.45 \pm 0.07) \mathrm{E}+1$ & $5.0 \mathrm{E}-1$ & $(1.23 \pm 0.18) \mathrm{E}-1$ \\
\hline 040803 & $15: 08: 57.750$ & $(1.14 \pm 0.11) \mathrm{E}+2$ & $1.5 \mathrm{E}+0$ & $-(3.95 \pm 1.05) \mathrm{E}-1$ \\
\hline 040810 & $14: 15: 42.650$ & $(1.92 \pm 0.05) \mathrm{E}+1$ & 3.0E-1 & $-(7.96 \pm 1.96) \mathrm{E}-2$ \\
\hline 040818 & $01: 29: 04.250$ & $(8.00 \pm 0.33) \mathrm{E}+0$ & $1.0 \mathrm{E}-1$ & $(4.57 \pm 0.42) \mathrm{E}-1$ \\
\hline 040822 & $21: 21: 55.125$ & $(1.38 \pm 0.14) \mathrm{E}+0$ & $3.0 \mathrm{E}-2$ & $(2.05 \pm 0.83) \mathrm{E}-1$ \\
\hline 040824 & 05:16:07.500 & $(4.90 \pm 0.50) \mathrm{E}+1$ & $1.0 \mathrm{E}+0$ & $-(4.90 \pm 7.92) \mathrm{E}-2$ \\
\hline 040921 & $16: 06: 20.265$ & $(2.80 \pm 0.73) \mathrm{E}-1$ & 7.0E-2 & $(1.88 \pm 0.64) \mathrm{E}-1$ \\
\hline 040925 & $22: 28: 59.750$ & $(4.75 \pm 0.16) E+1$ & $5.0 \mathrm{E}-1$ & $(6.66 \pm 2.68) \mathrm{E}-2$ \\
\hline 040926 & 04:03:18.100 & $(7.60 \pm 0.22) \mathrm{E}+0$ & $2.0 \mathrm{E}-1$ & $(1.93 \pm 0.11) \mathrm{E}-1$ \\
\hline 041003 & $09: 17: 56.250$ & $(9.00 \pm 0.74) \mathrm{E}+0$ & $5.0 \mathrm{E}-1$ & $-(1.16 \pm 0.52) \mathrm{E}-1$ \\
\hline 041006 & $12: 18: 40.350$ & $(1.11 \pm 0.08) \mathrm{E}+1$ & $3.0 \mathrm{E}-1$ & $-(4.71 \pm 0.80) \mathrm{E}-1$ \\
\hline 041007 & 02:02:08.350 & $(2.00 \pm 0.13) \mathrm{E}+0$ & $1.0 \mathrm{E}-1$ & $(3.52 \pm 0.37) \mathrm{E}-1$ \\
\hline 041009 & $06: 38: 21.250$ & $(7.50 \pm 0.76) \mathrm{E}+0$ & $3.0 \mathrm{E}-1$ & $-(8.60 \pm 7.65) \mathrm{E}-2$ \\
\hline 041010 & $00: 14: 57.555$ & $(2.50 \pm 0.22) \mathrm{E}-1$ & $1.0 \mathrm{E}-2$ & $(1.58 \pm 0.64) \mathrm{E}-1$ \\
\hline 041012 & $12: 40: 51.500$ & $(4.60 \pm 0.53) \mathrm{E}+1$ & $1.0 \mathrm{E}+0$ & $-(3.90 \pm 1.16) \mathrm{E}-1$ \\
\hline 041013A & $02: 35: 25.000$ & $(1.84 \pm 0.09) \mathrm{E}+2$ & $2.0 \mathrm{E}+0$ & $(1.27 \pm 3.84) \mathrm{E}-2$ \\
\hline 041013B & $22: 56: 27.865$ & $(3.60 \pm 0.46) \mathrm{E}-1$ & $3.0 \mathrm{E}-2$ & $(6.92 \pm 7.69) \mathrm{E}-2$ \\
\hline 041015 & $10: 22: 18.950$ & $(3.90 \pm 0.29) \mathrm{E}+0$ & $1.0 \mathrm{E}-1$ & $-(1.82 \pm 0.60) \mathrm{E}-1$ \\
\hline 041016 & $04: 39: 37.000$ & $(1.80 \pm 0.17) \mathrm{E}+1$ & $4.0 \mathrm{E}-1$ & $-(1.90 \pm 0.82) \mathrm{E}-1$ \\
\hline 041018 & $13: 08: 19.500$ & $(1.02 \pm 0.04) \mathrm{E}+2$ & $1.0 \mathrm{E}+0$ & $-(3.19 \pm 3.19) \mathrm{E}-2$ \\
\hline 041101 & $01: 49: 36.150$ & $(3.20 \pm 0.34) \mathrm{E}+0$ & $1.0 \mathrm{E}-1$ & $(2.28 \pm 0.85) \mathrm{E}-1$ \\
\hline 041102 & $11: 12: 23.750$ & $(2.70 \pm 0.22) \mathrm{E}+0$ & $1.0 \mathrm{E}-1$ & $(1.69 \pm 0.60) \mathrm{E}-1$ \\
\hline 041107 & $15: 49: 29.250$ & $(4.70 \pm 0.45) \mathrm{E}+1$ & $5.0 \mathrm{E}-1$ & $-(6.04 \pm 7.68) \mathrm{E}-2$ \\
\hline 041116 & $05: 34: 56.500$ & $(5.00 \pm 0.53) \mathrm{E}+1$ & $1.0 \mathrm{E}+0$ & $-(3.38 \pm 1.06) \mathrm{E}-1$ \\
\hline 041117 & $15: 18: 00.950$ & $(1.62 \pm 0.05) \mathrm{E}+1$ & $3.0 \mathrm{E}-1$ & $-(1.81 \pm 0.24) \mathrm{E}-1$ \\
\hline 041120 & $19: 23: 41.300$ & $(8.60 \pm 0.42) E+0$ & $2.0 \mathrm{E}-1$ & $-(3.20 \pm 0.44) \mathrm{E}-1$ \\
\hline 041125 & $16: 07: 27.400$ & $(2.52 \pm 0.04) \mathrm{E}+1$ & $4.0 \mathrm{E}-1$ & $-(2.34 \pm 0.06) \mathrm{E}-1$ \\
\hline 041202 & $02: 30: 57.300$ & $(1.66 \pm 0.03) \mathrm{E}+1$ & $2.0 \mathrm{E}-1$ & $(1.14 \pm 0.12) \mathrm{E}-1$ \\
\hline $041211 \mathrm{~A}$ & $07: 49: 56.450$ & $(2.06 \pm 0.05) \mathrm{E}+1$ & $1.0 \mathrm{E}-1$ & $-(1.33 \pm 0.23) \mathrm{E}-1$ \\
\hline $041211 \mathrm{~B}$ & $11: 31: 53.400$ & $(2.00 \pm 0.09) \mathrm{E}+1$ & $4.0 \mathrm{E}-1$ & $(8.20 \pm 3.24) \mathrm{E}-2$ \\
\hline $041211 \mathrm{C}$ & $23: 57: 42.925$ & $(6.15 \pm 0.16) \mathrm{E}+0$ & $1.5 \mathrm{E}-1$ & $(4.46 \pm 0.07) \mathrm{E}-1$ \\
\hline 041213 & $06: 59: 36.330$ & $(1.40 \pm 0.24) \mathrm{E}-1$ & 2.0E-2 & $(3.19 \pm 0.82) \mathrm{E}-1$ \\
\hline 041218 & $15: 45: 50.500$ & $(5.10 \pm 0.57) \mathrm{E}+1$ & $1.0 \mathrm{E}+0$ & $-(2.22 \pm 0.98) \mathrm{E}-1$ \\
\hline 041219 & $01: 42: 19.400$ & $(1.00 \pm 0.11) \mathrm{E}+1$ & $4.0 \mathrm{E}-1$ & $-(1.32 \pm 7.84) \mathrm{E}-2$ \\
\hline 041223 & $14: 06: 42.250$ & $(4.05 \pm 0.11) \mathrm{E}+1$ & $5.0 \mathrm{E}-1$ & $(8.55 \pm 2.11) \mathrm{E}-2$ \\
\hline 041224 & $20: 20: 58.250$ & $(3.85 \pm 0.58) \mathrm{E}+1$ & $5.0 \mathrm{E}-1$ & $-(3.21 \pm 1.42) \mathrm{E}-1$ \\
\hline 041231 & $21: 50: 48.050$ & $(1.00 \pm 0.12) \mathrm{E}+0$ & $1.0 \mathrm{E}-1$ & $(3.20 \pm 0.56) \mathrm{E}-1$ \\
\hline 050124 & 11:30:03.250 & $(2.80 \pm 0.38) \mathrm{E}+0$ & $1.0 \mathrm{E}-1$ & $-(2.24 \pm 1.12) \mathrm{E}-1$ \\
\hline 050126 & $21: 07: 37.700$ & $(3.30 \pm 0.10) E+1$ & $6.0 \mathrm{E}-1$ & $-(1.71 \pm 0.22) \mathrm{E}-1$ \\
\hline 050203 & $17: 22: 00.850$ & $(3.80 \pm 0.24) \mathrm{E}+0$ & $1.0 \mathrm{E}-1$ & $-(0.85 \pm 4.62) \mathrm{E}-2$ \\
\hline 050213 & $19: 24: 04.750$ & $(1.70 \pm 0.09) \mathrm{E}+1$ & $5.0 \mathrm{E}-1$ & $(2.43 \pm 0.37) \mathrm{E}-1$ \\
\hline 050214 & $11: 38: 33.500$ & $(4.00 \pm 0.66) \mathrm{E}+1$ & $1.0 \mathrm{E}+0$ & $-(0.16 \pm 1.23) \mathrm{E}-1$ \\
\hline 050216 & $07: 26: 34.275$ & $(5.00 \pm 0.72) \mathrm{E}-1$ & $5.0 \mathrm{E}-2$ & $(5.66 \pm 1.00) \mathrm{E}-1$ \\
\hline 050219 & $21: 05: 51.650$ & $(9.40 \pm 0.17) \mathrm{E}+0$ & $1.0 \mathrm{E}-1$ & $-(3.01 \pm 0.15) \mathrm{E}-1$ \\
\hline 050311 & $17: 06: 58.850$ & $(2.40 \pm 0.44) \mathrm{E}+0$ & $3.0 \mathrm{E}-1$ & $-(1.44 \pm 1.09) \mathrm{E}-1$ \\
\hline 050312 & $05: 40: 13.575$ & $(1.50 \pm 0.51) \mathrm{E}-1$ & $5.0 \mathrm{E}-2$ & $(3.35 \pm 0.82) \mathrm{E}-1$ \\
\hline 050314 & 08:33:08.900 & $(8.00 \pm 0.35) \mathrm{E}+0$ & $2.0 \mathrm{E}-1$ & $-(4.77 \pm 0.45) \mathrm{E}-1$ \\
\hline 050320 & 08:04:26.900 & $(1.54 \pm 0.13) E+1$ & $2.0 \mathrm{E}-1$ & $(5.51 \pm 6.52) \mathrm{E}-2$ \\
\hline 050321 & $22: 11: 51.500$ & $(7.00 \pm 0.75) \mathrm{E}+0$ & $2.0 \mathrm{E}-1$ & $-(8.21 \pm 8.41) \mathrm{E}-2$ \\
\hline 050326 & 09:53:56.500 & $(2.70 \pm 0.11) \mathrm{E}+1$ & $2.0 \mathrm{E}-1$ & $-(2.54 \pm 0.36) \mathrm{E}-1$ \\
\hline 050328 & $03: 25: 14.875$ & $(4.50 \pm 0.51) \mathrm{E}-1$ & $5.0 \mathrm{E}-2$ & $-(1.02 \pm 0.20) \mathrm{E}-1$ \\
\hline 050404 & $17: 27: 48.500$ & $(1.14 \pm 0.03) \mathrm{E}+1$ & $2.0 \mathrm{E}-1$ & $(1.03 \pm 0.18) \mathrm{E}-1$ \\
\hline 050409 & $01: 18: 36.050$ & $(1.26 \pm 0.05) \mathrm{E}+0$ & $2.0 \mathrm{E}-2$ & $(3.05 \pm 0.30) \mathrm{E}-1$ \\
\hline 050411 & 21:51:09.500 & $(6.80 \pm 0.69) \mathrm{E}+0$ & $2.0 \mathrm{E}-1$ & $-(1.18 \pm 0.80) \mathrm{E}-1$ \\
\hline 050412 & $18: 58: 45.900$ & $(1.94 \pm 0.08) \mathrm{E}+1$ & $2.0 \mathrm{E}-1$ & $-(5.03 \pm 0.51) \mathrm{E}-1$ \\
\hline 050429 & 14:09:50.900 & $(1.94 \pm 0.15) \mathrm{E}+1$ & $2.0 \mathrm{E}-1$ & $(1.35 \pm 0.63) \mathrm{E}-1$ \\
\hline 050430 & 09:13:09.100 & $(1.42 \pm 0.17) \mathrm{E}+1$ & $2.0 \mathrm{E}-1$ & $(0.20 \pm 9.23) \mathrm{E}-2$ \\
\hline 050501 & $08: 19: 38.825$ & $(2.40 \pm 0.19) \mathrm{E}+0$ & $1.5 \mathrm{E}-1$ & $(7.81 \pm 4.03) \mathrm{E}-2$ \\
\hline 050502 & $19: 56: 57.575$ & $(1.60 \pm 0.20) \mathrm{E}+0$ & $5.0 \mathrm{E}-2$ & $(1.61 \pm 0.97) \mathrm{E}-1$ \\
\hline 050509 & $09: 31: 26.700$ & $(2.00 \pm 0.04) \mathrm{E}+1$ & $2.0 \mathrm{E}-1$ & $-(6.35 \pm 1.57) \mathrm{E}-2$ \\
\hline 050516 & 12:58:05.200 & $(7.60 \pm 1.16) \mathrm{E}+0$ & $4.0 \mathrm{E}-1$ & $(1.16 \pm 1.11) \mathrm{E}-1$ \\
\hline
\end{tabular}

typical durations are similar to those obtained with the onedimensional analysis, the percentages being slightly different ( $\approx 86 \%$ long, $\approx 9 \%$ short, $\approx 5 \%$ intermediate). 
Table 7. continued.

\begin{tabular}{|c|c|c|c|c|}
\hline$\overline{\text { GRB }}$ & $\begin{array}{r}\text { Peak time } \\
\text { UTC }\end{array}$ & $\begin{array}{r}T_{90} \\
{[\mathrm{~s}]}\end{array}$ & $\begin{array}{r}\delta t_{\mathrm{res}} \\
{[\mathrm{s}]}\end{array}$ & $\begin{array}{r}\text { Hardness ratio } \\
\log H\end{array}$ \\
\hline $050525 \mathrm{~A}$ & $00: 02: 54.450$ & $(7.40 \pm 0.19) \mathrm{E}+0$ & $1.0 \mathrm{E}-1$ & $-(4.06 \pm 0.25) \mathrm{E}-1$ \\
\hline 050525B & 00:50:00.500 & $(1.26 \pm 0.03) \mathrm{E}+1$ & $2.0 \mathrm{E}-1$ & $(6.42 \pm 1.15) \mathrm{E}-2$ \\
\hline 050528 & $07: 05: 23.500$ & $(1.57 \pm 0.09) \mathrm{E}+2$ & $1.0 \mathrm{E}+0$ & $-(4.77 \pm 0.69) \mathrm{E}-1$ \\
\hline 050530 & $04: 44: 44.900$ & $(2.40 \pm 0.34) \mathrm{E}+0$ & $2.0 \mathrm{E}-1$ & $(4.69 \pm 1.10) \mathrm{E}-1$ \\
\hline 050531 & $04: 27: 26.700$ & $(3.32 \pm 0.05) \mathrm{E}+1$ & $2.0 \mathrm{E}-1$ & $-(8.44 \pm 1.34) \mathrm{E}-2$ \\
\hline 050614 & 12:02:00.500 & $(3.10 \pm 0.45) \mathrm{E}+1$ & $1.0 \mathrm{E}+0$ & $-(1.73 \pm 1.20) \mathrm{E}-1$ \\
\hline 050701 & $14: 22: 00.950$ & $(1.00 \pm 0.08) \mathrm{E}+1$ & $1.0 \mathrm{E}-1$ & $(2.54 \pm 0.69) \mathrm{E}-1$ \\
\hline 050702 & $07: 47: 44.160$ & $(1.12 \pm 0.16) \mathrm{E}+0$ & $8.0 \mathrm{E}-2$ & $(2.01 \pm 1.01) \mathrm{E}-1$ \\
\hline 050703 & $05: 31: 50.825$ & $(8.65 \pm 0.44) \mathrm{E}+0$ & $5.0 \mathrm{E}-2$ & $-(2.24 \pm 0.47) \mathrm{E}-1$ \\
\hline 050706 & $17: 11: 37.875$ & $(6.15 \pm 0.42) \mathrm{E}+0$ & $1.5 \mathrm{E}-1$ & $-(0.07 \pm 5.20) \mathrm{E}-2$ \\
\hline 050713A & $04: 29: 11.750$ & $(1.86 \pm 0.07) \mathrm{E}+1$ & $3.0 \mathrm{E}-1$ & $-(9.16 \pm 2.81) \mathrm{E}-2$ \\
\hline 050713B & $12: 07: 28.750$ & $(6.05 \pm 0.39) \mathrm{E}+1$ & $5.0 \mathrm{E}-1$ & $-(0.35 \pm 5.23) \mathrm{E}-2$ \\
\hline 050715 & $01: 15: 49.250$ & $(1.14 \pm 0.03) \mathrm{E}+1$ & $1.0 \mathrm{E}-1$ & $-(2.32 \pm 0.20) \mathrm{E}-1$ \\
\hline 050717 & 10:30:55.100 & $(9.60 \pm 0.32) \mathrm{E}+0$ & $2.0 \mathrm{E}-1$ & $(2.36 \pm 0.23) \mathrm{E}-1$ \\
\hline 050726 & $20: 22: 19.800$ & $(1.60 \pm 0.06) \mathrm{E}+1$ & $4.0 \mathrm{E}-1$ & $-(5.78 \pm 2.30) \mathrm{E}-2$ \\
\hline 050729 & 01:09:41.120 & $(4.40 \pm 0.47) \mathrm{E}+0$ & $8.0 \mathrm{E}-2$ & $-(8.49 \pm 8.47) \mathrm{E}-2$ \\
\hline 050802 & 10:08:02.850 & $(2.94 \pm 0.31) \mathrm{E}+1$ & $3.0 \mathrm{E}-1$ & $-(1.76 \pm 0.90) \mathrm{E}-1$ \\
\hline 050805 & $13: 29: 47.625$ & $(1.05 \pm 0.08) \mathrm{E}+0$ & $5.0 \mathrm{E}-2$ & $(2.99 \pm 0.53) \mathrm{E}-1$ \\
\hline 050809 & $20: 15: 26.720$ & $(2.40 \pm 0.14) \mathrm{E}+0$ & $8.0 \mathrm{E}-2$ & $-(7.56 \pm 3.92) \mathrm{E}-2$ \\
\hline 050813 & $21: 13: 43.900$ & $(8.00 \pm 0.87) \mathrm{E}+0$ & $2.0 \mathrm{E}-1$ & $(3.68 \pm 0.99) \mathrm{E}-1$ \\
\hline 050814 & $04: 35: 19.451$ & $(1.24 \pm 0.08) \mathrm{E}-1$ & $2.0 \mathrm{E}-3$ & $(6.01 \pm 0.64) \mathrm{E}-1$ \\
\hline 050817 & $10: 43: 18.900$ & $(2.12 \pm 0.20) \mathrm{E}+1$ & $2.0 \mathrm{E}-1$ & $(3.69 \pm 0.86) \mathrm{E}-1$ \\
\hline 050820 & $23: 50: 36.050$ & $(6.00 \pm 0.58) \mathrm{E}+0$ & $3.0 \mathrm{E}-1$ & $-(3.57 \pm 0.87) \mathrm{E}-1$ \\
\hline 050824 & $11: 57: 42.535$ & $(2.50 \pm 0.14) \mathrm{E}-1$ & $1.0 \mathrm{E}-2$ & $(3.31 \pm 0.35) \mathrm{E}-1$ \\
\hline 050825 & $03: 34: 27.850$ & $(8.00 \pm 0.44) \mathrm{E}-1$ & $2.0 \mathrm{E}-2$ & $(1.62 \pm 0.42) \mathrm{E}-1$ \\
\hline 050902 & $12: 24: 30.650$ & $(1.16 \pm 0.10) \mathrm{E}+1$ & $1.0 \mathrm{E}-1$ & $-(5.97 \pm 1.18) \mathrm{E}-1$ \\
\hline 050923 & $01: 37: 44.850$ & $(9.60 \pm 0.56) \mathrm{E}+0$ & $1.0 \mathrm{E}-1$ & $(6.55 \pm 4.65) \mathrm{E}-2$ \\
\hline 051009 & $10: 49: 02.750$ & $(8.50 \pm 0.52) \mathrm{E}+1$ & $5.0 \mathrm{E}-1$ & $-(2.22 \pm 0.57) \mathrm{E}-1$ \\
\hline 051012 & $12: 00: 11.925$ & $(2.55 \pm 0.08) \mathrm{E}+0$ & $5.0 \mathrm{E}-2$ & $(1.14 \pm 1.98) \mathrm{E}-2$ \\
\hline 051021 & $14: 01: 14.625$ & $(5.10 \pm 0.10) \mathrm{E}+1$ & $2.5 \mathrm{E}-1$ & $-(2.26 \pm 0.17) \mathrm{E}-1$ \\
\hline 051031 & 22:01:05.750 & $(4.95 \pm 0.28) \mathrm{E}+1$ & $1.5 \mathrm{E}+0$ & $(4.49 \pm 3.88) \mathrm{E}-2$ \\
\hline 051101 & $01: 13: 12.625$ & $(1.10 \pm 0.14) \mathrm{E}+1$ & $1.5 \mathrm{E}-1$ & $(9.19 \pm 9.99) \mathrm{E}-2$ \\
\hline 051103 & $09: 25: 42.192$ & $(1.40 \pm 0.05) \mathrm{E}-1$ & $5.0 \mathrm{E}-3$ & $(4.68 \pm 0.15) \mathrm{E}-1$ \\
\hline 051109 & $16: 42: 00.750$ & $(2.55 \pm 0.16) \mathrm{E}+1$ & $5.0 \mathrm{E}-1$ & $-(2.15 \pm 0.58) \mathrm{E}-1$ \\
\hline 051111 & 05:59:40.100 & $(2.38 \pm 0.15) \mathrm{E}+1$ & $2.0 \mathrm{E}-1$ & $(1.10 \pm 0.53) \mathrm{E}-1$ \\
\hline 051117 & $12: 34: 25.300$ & $(3.32 \pm 0.13) \mathrm{E}+1$ & $2.0 \mathrm{E}-1$ & $(1.62 \pm 0.34) \mathrm{E}-1$ \\
\hline 051119 & $13: 10: 59.900$ & $(3.78 \pm 0.31) \mathrm{E}+1$ & $2.0 \mathrm{E}-1$ & $-(1.74 \pm 0.73) \mathrm{E}-1$ \\
\hline 051124A & $08: 16: 59.450$ & $(1.92 \pm 0.15) \mathrm{E}+1$ & $1.0 \mathrm{E}-1$ & $(2.31 \pm 0.65) \mathrm{E}-1$ \\
\hline 051124B & $14: 20: 11.100$ & $(3.68 \pm 0.19) \mathrm{E}+1$ & $2.0 \mathrm{E}-1$ & $-(2.16 \pm 0.48) \mathrm{E}-1$ \\
\hline 051201A & $18: 31: 37.250$ & $(2.25 \pm 0.29) \mathrm{E}+1$ & $1.5 \mathrm{E}+0$ & $-(0.50 \pm 8.84) \mathrm{E}-2$ \\
\hline 051201B & $22: 35: 30.350$ & $(1.84 \pm 0.13) \mathrm{E}+1$ & $1.0 \mathrm{E}-1$ & $(1.29 \pm 0.59) \mathrm{E}-1$ \\
\hline 051207 & 19:04:09.350 & $(5.73 \pm 0.17) \mathrm{E}+1$ & $3.0 \mathrm{E}-1$ & $-(1.75 \pm 0.27) \mathrm{E}-1$ \\
\hline 051211 & $05: 28: 13.250$ & $(2.94 \pm 0.09) \mathrm{E}+1$ & $7.0 \mathrm{E}-1$ & $(1.24 \pm 0.17) \mathrm{E}-1$ \\
\hline 051217 & 09:54:08.650 & $(1.80 \pm 0.27) \mathrm{E}+1$ & $3.0 \mathrm{E}-1$ & $-(0.28 \pm 1.14) \mathrm{E}-1$ \\
\hline 051220 & 13:04:17.525 & $(1.29 \pm 0.01) \mathrm{E}+1$ & $5.0 \mathrm{E}-2$ & $(2.15 \pm 0.04) \mathrm{E}-1$ \\
\hline 051220 & $21: 34: 36.750$ & $(2.80 \pm 0.46) \mathrm{E}+1$ & $5.0 \mathrm{E}-1$ & $-(5.31 \pm 2.02) \mathrm{E}-1$ \\
\hline 051221 & 01:51:15.975 & $(2.80 \pm 0.17) \mathrm{E}-1$ & $1.0 \mathrm{E}-2$ & $(1.65 \pm 0.41) \mathrm{E}-1$ \\
\hline 051222 & $15: 07: 35.600$ & $(2.44 \pm 0.40) \mathrm{E}+1$ & $4.0 \mathrm{E}-1$ & $-(1.73 \pm 1.35) \mathrm{E}-1$ \\
\hline 060101 & $00: 34: 11.800$ & $(1.96 \pm 0.09) \mathrm{E}+1$ & $4.0 \mathrm{E}-1$ & $(6.61 \pm 3.35) \mathrm{E}-2$ \\
\hline 060110 & 08:01:18.900 & $(1.20 \pm 0.16) \mathrm{E}+1$ & $2.0 \mathrm{E}-1$ & $(1.03 \pm 1.00) \mathrm{E}-1$ \\
\hline 060111 & 08:49:00.250 & $(6.90 \pm 0.25) \mathrm{E}+1$ & $1.5 \mathrm{E}+0$ & $(1.61 \pm 0.24) \mathrm{E}-1$ \\
\hline 060117 & $06: 50: 13.850$ & $(1.59 \pm 0.10) \mathrm{E}+1$ & $3.0 \mathrm{E}-1$ & $-(3.13 \pm 0.63) \mathrm{E}-1$ \\
\hline 060121A & $04: 12: 56.500$ & $(4.40 \pm 0.63) \mathrm{E}+1$ & $1.0 \mathrm{E}+0$ & $(0.17 \pm 1.08) \mathrm{E}-1$ \\
\hline 060121B & $22: 24: 56.755$ & $(2.38 \pm 0.12) \mathrm{E}+0$ & $7.0 \mathrm{E}-2$ & $-(1.05 \pm 0.36) \mathrm{E}-1$ \\
\hline 060123 & 05:05:24.900 & $(1.10 \pm 0.03) \mathrm{E}+1$ & $2.0 \mathrm{E}-1$ & $-(1.31 \pm 0.17) \mathrm{E}-1$ \\
\hline 060124 & $16: 04: 22.400$ & $(1.76 \pm 0.13) \mathrm{E}+1$ & $4.0 \mathrm{E}-1$ & $(4.99 \pm 5.58) \mathrm{E}-2$ \\
\hline 060130 & $13: 48: 31.100$ & $(1.52 \pm 0.08) \mathrm{E}+1$ & $2.0 \mathrm{E}-1$ & $(2.98 \pm 4.17) \mathrm{E}-2$ \\
\hline 060203 & $07: 28: 58.535$ & $(5.40 \pm 0.45) \mathrm{E}-1$ & $1.0 \mathrm{E}-2$ & $(1.43 \pm 0.66) \mathrm{E}-1$ \\
\hline 060217 & $09: 47: 43.325$ & $(1.91 \pm 0.08) \mathrm{E}+1$ & $1.5 \mathrm{E}-1$ & $-(7.34 \pm 3.62) \mathrm{E}-2$ \\
\hline 060224 & 02:31:11.500 & $(1.00 \pm 0.06) \mathrm{E}+1$ & $2.0 \mathrm{E}-1$ & $-(8.14 \pm 4.54) \mathrm{E}-2$ \\
\hline 060228 & $03: 17: 33.850$ & $(3.27 \pm 0.21) \mathrm{E}+1$ & $3.0 \mathrm{E}-1$ & $-(3.88 \pm 5.28) \mathrm{E}-2$ \\
\hline 060303 & $22: 42: 47.525$ & $(5.00 \pm 0.53) \mathrm{E}-1$ & $5.0 \mathrm{E}-2$ & $(2.56 \pm 0.29) \mathrm{E}-1$ \\
\hline 060306 & $15: 22: 38.485$ & $(9.20 \pm 0.11) \mathrm{E}-1$ & $1.0 \mathrm{E}-2$ & $-(1.07 \pm 0.04) \mathrm{E}-1$ \\
\hline 060309 & $14: 38: 54.250$ & $(2.95 \pm 0.30) E+1$ & $5.0 \mathrm{E}-1$ & $(1.32 \pm 0.81) \mathrm{E}-1$ \\
\hline 060312A & $06: 17: 21.115$ & $(2.40 \pm 0.47) \mathrm{E}-1$ & $3.0 \mathrm{E}-2$ & $(2.48 \pm 1.16) \mathrm{E}-1$ \\
\hline 060312B & $16: 44: 53.400$ & $(6.80 \pm 1.18) \mathrm{E}+0$ & $4.0 \mathrm{E}-1$ & $-(2.32 \pm 1.40) \mathrm{E}-1$ \\
\hline
\end{tabular}

Three classes of GRBs have also been identified for the BATSE GRBs (Horváth et al. 2006) and the Swift GRBs (Horváth et al. 2008). For BATSE, $\approx 65 \%$ of all GRBs are long, $\approx 24 \%$ short, and $\approx 11 \%$ intermediate (Horváth et al. 2006,
Table 7. continued.

\begin{tabular}{|c|c|c|c|c|}
\hline GRB & $\begin{array}{r}\text { Peak time } \\
\text { UTC }\end{array}$ & $\begin{array}{r}T_{90} \\
{[\mathrm{~s}]}\end{array}$ & $\begin{array}{r}\delta t_{\mathrm{res}} \\
{[\mathrm{s}]}\end{array}$ & $\begin{array}{r}\text { Hardness ratio } \\
\log H\end{array}$ \\
\hline 060313 & $20: 11: 32.900$ & $(3.60 \pm 0.51) \mathrm{E}+0$ & $2.0 \mathrm{E}-1$ & $(1.52 \pm 1.03) \mathrm{E}-1$ \\
\hline 060323 & $07: 04: 30.100$ & $(1.76 \pm 0.04) E+1$ & $2.0 \mathrm{E}-1$ & $(3.37 \pm 1.52) \mathrm{E}-2$ \\
\hline 060325 & $12: 02: 20.350$ & $(1.08 \pm 0.02) \mathrm{E}+1$ & $1.0 \mathrm{E}-1$ & $(0.70 \pm 1.11) \mathrm{E}-2$ \\
\hline 060401 & $05: 40: 18.750$ & $(6.30 \pm 0.17) \mathrm{E}+0$ & $1.0 \mathrm{E}-1$ & $(9.21 \pm 1.86) \mathrm{E}-2$ \\
\hline 060408 & $13: 11: 39.150$ & $(6.90 \pm 0.95) \mathrm{E}+0$ & 3.0E-1 & $-(0.06 \pm 1.01) \mathrm{E}-1$ \\
\hline 060415 & 05:31:00.050 & $(1.32 \pm 0.20) \mathrm{E}+1$ & $3.0 \mathrm{E}-1$ & $(1.80 \pm 1.14) \mathrm{E}-1$ \\
\hline 060418 & 03:06:35.800 & $(4.08 \pm 0.21) \mathrm{E}+1$ & $4.0 \mathrm{E}-1$ & $-(1.23 \pm 0.43) \mathrm{E}-1$ \\
\hline $060421 \mathrm{~A}$ & 11:03:49.000 & $(3.12 \pm 0.11) \mathrm{E}+1$ & $4.0 \mathrm{E}-1$ & $-(8.58 \pm 2.76) \mathrm{E}-2$ \\
\hline 060421B & $20: 36: 38.200$ & $(2.16 \pm 0.19) \mathrm{E}+1$ & 4.0E-1 & $-(1.39 \pm 0.74) \mathrm{E}-1$ \\
\hline 060425 & $16: 57: 38.705$ & $(1.40 \pm 0.12) \mathrm{E}-1$ & $1.0 \mathrm{E}-2$ & $(2.59 \pm 0.42) \mathrm{E}-1$ \\
\hline 060428 & $02: 30: 41.750$ & $(1.35 \pm 0.23) \mathrm{E}+1$ & $5.0 \mathrm{E}-1$ & $-(2.45 \pm 1.44) \mathrm{E}-1$ \\
\hline 060429 & $12: 19: 51.250$ & $(2.00 \pm 0.21) \mathrm{E}-1$ & $2.0 \mathrm{E}-2$ & $(2.89 \pm 0.33) \mathrm{E}-1$ \\
\hline 060505 & $23: 32: 01.050$ & $(9.60 \pm 0.46) \mathrm{E}+0$ & $3.0 \mathrm{E}-1$ & $(2.15 \pm 0.30) \mathrm{E}-1$ \\
\hline 060528 & $22: 53: 05.750$ & $(7.70 \pm 0.20) \mathrm{E}+1$ & $5.0 \mathrm{E}-1$ & $(2.41 \pm 0.21) \mathrm{E}-1$ \\
\hline 060530 & 19:19:11.300 & $(4.00 \pm 0.35) \mathrm{E}+0$ & $2.0 \mathrm{E}-1$ & $-(1.16 \pm 0.63) \mathrm{E}-1$ \\
\hline 060610 & $11: 22: 24.070$ & $(6.00 \pm 0.33) \mathrm{E}-1$ & 2.0E-2 & $(2.48 \pm 0.38) \mathrm{E}-1$ \\
\hline 060614 & $12: 43: 48.250$ & $(5.25 \pm 0.45) \mathrm{E}+1$ & $1.5 \mathrm{E}+0$ & $-(1.78 \pm 0.72) \mathrm{E}-1$ \\
\hline 060622 & $17: 19: 48.750$ & $(2.35 \pm 0.09) \mathrm{E}+1$ & $5.0 \mathrm{E}-1$ & $(1.12 \pm 0.27) \mathrm{E}-1$ \\
\hline 060624 & $13: 46: 56.255$ & $(2.55 \pm 0.07) \mathrm{E}+0$ & $3.0 \mathrm{E}-2$ & $-(4.55 \pm 0.29) \mathrm{E}-1$ \\
\hline 060625 & $07: 33: 27.100$ & $(4.40 \pm 0.37) \mathrm{E}+0$ & $2.0 \mathrm{E}-1$ & $(2.21 \pm 0.58) \mathrm{E}-1$ \\
\hline 060630 & 00:06:41.250 & $(4.10 \pm 0.19) \mathrm{E}+1$ & $5.0 \mathrm{E}-1$ & $-(9.96 \pm 4.00) \mathrm{E}-2$ \\
\hline 060708 & $04: 30: 38.485$ & $(1.14 \pm 0.08) \mathrm{E}-1$ & $6.0 \mathrm{E}-3$ & $(1.57 \pm 0.36) \mathrm{E}-1$ \\
\hline 060729 & 04:07:38.600 & $(5.20 \pm 0.53) \mathrm{E}+0$ & $2.0 \mathrm{E}-1$ & $-(1.16 \pm 0.79) \mathrm{E}-1$ \\
\hline 060805 & $14: 27: 17.450$ & $(5.10 \pm 0.07) \mathrm{E}+0$ & $6.0 \mathrm{E}-2$ & $(6.98 \pm 0.69) \mathrm{E}-2$ \\
\hline 060811 & $16: 56: 43.950$ & $(7.29 \pm 0.27) \mathrm{E}+1$ & $3.0 \mathrm{E}-1$ & $-(1.96 \pm 0.35) \mathrm{E}-1$ \\
\hline 060819 & $18: 28: 20.700$ & $(2.40 \pm 0.11) \mathrm{E}+1$ & $6.0 \mathrm{E}-1$ & $(0.84 \pm 3.26) \mathrm{E}-2$ \\
\hline 060823 & $08: 05: 33.750$ & $(1.00 \pm 0.18) \mathrm{E}+0$ & $1.0 \mathrm{E}-1$ & $(3.23 \pm 1.26) \mathrm{E}-1$ \\
\hline 060919 & $21: 52: 12.750$ & $(2.95 \pm 0.37) \mathrm{E}+1$ & $5.0 \mathrm{E}-1$ & $(1.20 \pm 0.95) \mathrm{E}-1$ \\
\hline 060920 & $15: 32: 38.700$ & $(2.18 \pm 0.03) \mathrm{E}+1$ & $2.0 \mathrm{E}-1$ & $(3.59 \pm 1.01) \mathrm{E}-2$ \\
\hline 060925 & $20: 14: 35.375$ & $(1.65 \pm 0.08) \mathrm{E}+1$ & $2.5 \mathrm{E}-1$ & $-(1.00 \pm 0.38) \mathrm{E}-1$ \\
\hline 060928 & $01: 20: 24.150$ & $(2.03 \pm 0.03) \mathrm{E}+2$ & $3.0 \mathrm{E}-1$ & $-(1.28 \pm 1.19) \mathrm{E}-2$ \\
\hline 061005 & $13: 38: 01.800$ & $(4.24 \pm 0.12) \mathrm{E}+1$ & $4.0 \mathrm{E}-1$ & $-(2.05 \pm 0.25) \mathrm{E}-1$ \\
\hline 061006A & $08: 43: 39.225$ & $(1.65 \pm 0.10) \mathrm{E}+0$ & $5.0 \mathrm{E}-2$ & $(1.53 \pm 0.43) \mathrm{E}-1$ \\
\hline 061006B & $16: 45: 27.875$ & $(4.00 \pm 0.53) \mathrm{E}-1$ & $5.0 \mathrm{E}-2$ & $(3.23 \pm 0.39) \mathrm{E}-1$ \\
\hline 061007 & $10: 08: 54.150$ & $(6.06 \pm 0.07) \mathrm{E}+1$ & $3.0 \mathrm{E}-1$ & $(1.20 \pm 0.08) \mathrm{E}-1$ \\
\hline 061012 & $11: 51: 57.850$ & $(9.30 \pm 0.48) \mathrm{E}+0$ & $1.0 \mathrm{E}-1$ & $(2.56 \pm 0.44) \mathrm{E}-1$ \\
\hline 061013 & 18:06:28.200 & $(4.00 \pm 0.22) \mathrm{E}+1$ & $8.0 \mathrm{E}-1$ & $-(1.54 \pm 0.45) \mathrm{E}-1$ \\
\hline 061014 & $06: 17: 02.375$ & $(2.00 \pm 0.55) \mathrm{E}-1$ & $5.0 \mathrm{E}-2$ & $(2.56 \pm 0.94) \mathrm{E}-1$ \\
\hline 061022 & $12: 23: 42.850$ & $(2.19 \pm 0.29) \mathrm{E}+1$ & $3.0 \mathrm{E}-1$ & $-(0.44 \pm 1.02) \mathrm{E}-1$ \\
\hline 061031 & $12: 19: 51.500$ & $(3.30 \pm 0.12) E+1$ & $2.0 \mathrm{E}-1$ & $(7.92 \pm 3.06) \mathrm{E}-2$ \\
\hline 061101 & $21: 26: 51.550$ & $(1.92 \pm 0.30) \mathrm{E}+1$ & $3.0 \mathrm{E}-1$ & $-(2.41 \pm 1.40) \mathrm{E}-1$ \\
\hline 061108 & $01: 09: 54.850$ & $(3.75 \pm 0.09) \mathrm{E}+1$ & $3.0 \mathrm{E}-1$ & $-(8.68 \pm 1.90) \mathrm{E}-2$ \\
\hline 061113 & $13: 43: 36.050$ & $(1.82 \pm 0.03) \mathrm{E}+1$ & $1.0 \mathrm{E}-1$ & $(1.76 \pm 0.13) \mathrm{E}-1$ \\
\hline 061117 & $06: 00: 11.250$ & $(2.15 \pm 0.27) \mathrm{E}+1$ & $5.0 \mathrm{E}-1$ & $(9.86 \pm 9.49) \mathrm{E}-2$ \\
\hline 061121 & $15: 23: 44.275$ & $(1.46 \pm 0.03) \mathrm{E}+1$ & $1.5 \mathrm{E}-1$ & $(1.27 \pm 0.17) \mathrm{E}-1$ \\
\hline 061123 & $16: 33: 28.650$ & $(5.70 \pm 0.32) \mathrm{E}+0$ & $1.0 \mathrm{E}-1$ & $(1.23 \pm 0.43) \mathrm{E}-1$ \\
\hline 061126 & 08:48:03.150 & $(1.65 \pm 0.07) \mathrm{E}+1$ & $3.0 \mathrm{E}-1$ & $(2.49 \pm 0.31) \mathrm{E}-1$ \\
\hline 061128 & 20:01:11.805 & $(3.00 \pm 0.31) \mathrm{E}-1$ & $3.0 \mathrm{E}-2$ & $(3.50 \pm 0.27) \mathrm{E}-1$ \\
\hline 061205 & $05: 22: 15.450$ & $(7.50 \pm 0.91) \mathrm{E}+0$ & $3.0 \mathrm{E}-1$ & $-(1.22 \pm 0.96) \mathrm{E}-1$ \\
\hline 061212 & $05: 31: 30.970$ & $(1.90 \pm 0.01) \mathrm{E}+1$ & $6.0 \mathrm{E}-2$ & $(2.73 \pm 0.06) \mathrm{E}-1$ \\
\hline 061222 & $03: 30: 19.300$ & $(1.16 \pm 0.05) \mathrm{E}+1$ & $2.0 \mathrm{E}-1$ & $(1.44 \pm 0.31) \mathrm{E}-1$ \\
\hline 061229 & $22: 25: 44.250$ & $(7.95 \pm 0.97) \mathrm{E}+1$ & $5.0 \mathrm{E}-1$ & $(1.69 \pm 0.93) \mathrm{E}-1$ \\
\hline 061230 & $23: 09: 31.000$ & $(2.56 \pm 0.19) \mathrm{E}+1$ & $8.0 \mathrm{E}-1$ & $-(1.63 \pm 0.60) \mathrm{E}-1$ \\
\hline 070113 & $11: 56: 23.815$ & $(2.70 \pm 0.48) \mathrm{E}-1$ & $3.0 \mathrm{E}-2$ & $(1.65 \pm 1.04) \mathrm{E}-1$ \\
\hline 070116 & $14: 32: 16.125$ & $(1.65 \pm 0.11) \mathrm{E}+1$ & $2.5 \mathrm{E}-1$ & $(3.79 \pm 5.24) \mathrm{E}-2$ \\
\hline 070120 & $10: 48: 36.250$ & $(1.85 \pm 0.27) \mathrm{E}+1$ & $5.0 \mathrm{E}-1$ & $(1.94 \pm 1.07) \mathrm{E}-1$ \\
\hline 070121 & $10: 12: 17.000$ & $(8.80 \pm 1.60) \mathrm{E}+0$ & $8.0 \mathrm{E}-1$ & $(0.11 \pm 1.19) \mathrm{E}-1$ \\
\hline 070125 & $07: 21: 27.250$ & $(5.67 \pm 0.08) \mathrm{E}+1$ & $3.0 \mathrm{E}-1$ & $(5.03 \pm 1.12) \mathrm{E}-2$ \\
\hline 070214 & $22: 39: 20.850$ & $(1.77 \pm 0.27) \mathrm{E}+1$ & $3.0 \mathrm{E}-1$ & $(1.00 \pm 1.11) \mathrm{E}-1$ \\
\hline 070220 & 04:44:45.300 & $(2.14 \pm 0.11) \mathrm{E}+1$ & $2.0 \mathrm{E}-1$ & $(4.86 \pm 4.03) \mathrm{E}-2$ \\
\hline 070221 & 21:06:46.500 & $(1.02 \pm 0.13) \mathrm{E}+1$ & 2.0E-1 & $-(2.28 \pm 9.64) \mathrm{E}-2$ \\
\hline 070307 & $21: 15: 43.250$ & $(5.25 \pm 0.27) \mathrm{E}+1$ & $5.0 \mathrm{E}-1$ & $-(5.80 \pm 4.35) \mathrm{E}-2$ \\
\hline 070402 & $15: 48: 39.475$ & $(8.85 \pm 0.56) \mathrm{E}+0$ & $1.5 \mathrm{E}-1$ & $(4.96 \pm 4.92) \mathrm{E}-2$ \\
\hline 070420 & $06: 18: 18.400$ & $(6.16 \pm 0.36) \mathrm{E}+1$ & $8.0 \mathrm{E}-1$ & $-(1.63 \pm 0.51) \mathrm{E}-1$ \\
\hline 070508 & $04: 18: 25.050$ & $(1.31 \pm 0.04) \mathrm{E}+1$ & $1.0 \mathrm{E}-1$ & $-(1.71 \pm 2.74) \mathrm{E}-2$ \\
\hline 070516 & $20: 41: 24.725$ & $(3.50 \pm 0.56) \mathrm{E}-1$ & $5.0 \mathrm{E}-2$ & $(5.01 \pm 0.73) \mathrm{E}-1$ \\
\hline
\end{tabular}

Table 2 of that article). The typical durations found for BATSE are roughly a factor of 2 longer than for RHESSI, but consistent for all three classes. As is known from BATSE, also in the RHESSI data set, the short GRBs are on average harder than the 
Table 7. continued.

\begin{tabular}{|c|c|c|c|c|}
\hline GRB & $\begin{array}{r}\text { Peak time } \\
\text { UTC }\end{array}$ & $\begin{array}{r}T_{90} \\
{[\mathrm{~s}]}\end{array}$ & $\begin{array}{r}\delta t_{\text {res }} \\
{[\mathrm{s}]}\end{array}$ & $\begin{array}{r}\text { Hardness ratio } \\
\log H\end{array}$ \\
\hline 070531 & 11:45:43.500 & $(2.60 \pm 0.34) \mathrm{E}+1$ & $1.0 \mathrm{E}+0$ & $-(1.08 \pm 1.03) \mathrm{E}-1$ \\
\hline 070614 & 05:05:09.425 & $(1.50 \pm 0.51) \mathrm{E}-1$ & $5.0 \mathrm{E}-2$ & $.64) \mathrm{E}-1$ \\
\hline 070622 & $02: 25: 17.850$ & $(1.38 \pm 0.03) \mathrm{E}+1$ & $1.0 \mathrm{E}-1$ & $(1.23 \pm 0.18) \mathrm{E}-1$ \\
\hline 070626 & 04:08:44.500 & $(1.43 \pm 0.02) \mathrm{E}+2$ & $1.0 \mathrm{E}+0$ & $(5.00 \pm 1.07) \mathrm{E}-2$ \\
\hline 070710 & 08:22:07.850 & $(3.90 \pm 0.58) \mathrm{E}+0$ & $3.0 \mathrm{E}-1$ & .91) $\mathrm{E}-2$ \\
\hline 070717 & $21: 50: 38.750$ & $(1.75 \pm 0.20) \mathrm{E}+1$ & $5.0 \mathrm{E}-1$ & $(2.50 \pm 0.87) \mathrm{E}-1$ \\
\hline 070722 & 06:00:31.500 & $(7.20 \pm 0.96) \mathrm{E}+0$ & $2.0 \mathrm{E}-1$ & $(1.02 \pm 0.99) \mathrm{E}-1$ \\
\hline 070724 & $23: 25: 46.500$ & $(2.40 \pm 0.25) \mathrm{E}+1$ & $1.0 \mathrm{E}+0$ & $-(2.08 \pm 0.89) \mathrm{E}-1$ \\
\hline 070802 & 06:16:19.390 & $(3.12 \pm 0.25) \mathrm{E}+0$ & $6.0 \mathrm{E}-2$ & $(4.80 \pm 0.78) \mathrm{E}-1$ \\
\hline 070817 & $14: 43: 42.250$ & $(8.80 \pm 0.71) \mathrm{E}+1$ & $5.0 \mathrm{E}-1$ & $-(9.18 \pm 6.84) \mathrm{E}-2$ \\
\hline 070819 & 10:17:04.750 & $(3.30 \pm 0.26) \mathrm{E}+1$ & $5.0 \mathrm{E}-1$ & $(3.83 \pm 6.10) \mathrm{E}-2$ \\
\hline 070821 & $12: 51: 31.750$ & $(6.75 \pm 0.21) \mathrm{E}+1$ & $5.0 \mathrm{E}-1$ & $(6.03 \pm 2.47) \mathrm{E}-2$ \\
\hline 070824 & 20:50:10.425 & $(1.30 \pm 0.07) \mathrm{E}+0$ & $5.0 \mathrm{E}-2$ & $(1.01 \pm 0.29) \mathrm{E}-1$ \\
\hline 070825 & $01: 55: 54.250$ & $(3.42 \pm 0.09) \mathrm{E}+1$ & 3.0E-1 & $(1.44 \pm 0.19) \mathrm{E}-1$ \\
\hline 070917 & 09:40:31.250 & $(2.31 \pm 0.24) \mathrm{E}+1$ & $3.0 \mathrm{E}-1$ & $(3.20 \pm 0.85) \mathrm{E}-1$ \\
\hline 071013 & 08:53:39.475 & $(3.65 \pm 0.43) \mathrm{E}+0$ & $5.0 \mathrm{E}-2$ & $(3.27 \pm 8.94) \mathrm{E}-2$ \\
\hline 071014 & 03:19:52.450 & $(7.80 \pm 0.37) \mathrm{E}+0$ & $1.0 \mathrm{E}-1$ & $(4.13 \pm 3.71) \mathrm{E}-2$ \\
\hline 071030 & 08:52:41.900 & $(6.00 \pm 0.91) \mathrm{E}+0$ & $2.0 \mathrm{E}-1$ & $(2.20 \pm 1.14) \mathrm{E}-1$ \\
\hline 071104 & 11:41:09.525 & $(1.47 \pm 0.13) \mathrm{E}+1$ & $1.5 \mathrm{E}-1$ & $-(6.66 \pm 7.12) \mathrm{E}-2$ \\
\hline 071204 & 05:58:29.475 & $(3.00 \pm 0.56) \mathrm{E}-1$ & $5.0 \mathrm{E}-2$ & $(3.46 \pm 0.72) \mathrm{E}-1$ \\
\hline 071217 & $17: 03: 27.950$ & $(8.30 \pm 0.48) \mathrm{E}+0$ & $1.0 \mathrm{E}-1$ & $-(9.40 \pm 4.89) \mathrm{E}-2$ \\
\hline 080114 & $16: 10: 22.300$ & $(7.34 \pm 0.07) \mathrm{E}+1$ & $2.0 \mathrm{E}-1$ & $(3.60 \pm 0.09) \mathrm{E}-1$ \\
\hline 080202 & 13:04:37.250 & $(3.06 \pm 0.39) \mathrm{E}+1$ & $3.0 \mathrm{E}-1$ & $(0.15 \pm 9.87)$ E- 2 \\
\hline 080204 & $13: 56: 34.760$ & $(4.88 \pm 0.23) \mathrm{E}+0$ & $8.0 \mathrm{E}-2$ & $(4.53 \pm 0.42) \mathrm{E}-1$ \\
\hline 080211 & $07: 23: 46.250$ & $(2.80 \pm 0.08) \mathrm{E}+1$ & $5.0 \mathrm{E}-1$ & $(2.79 \pm 0.19) \mathrm{E}-1$ \\
\hline 080218 & 05:57:28.375 & $(1.95 \pm 0.15) \mathrm{E}+1$ & $2.5 \mathrm{E}-1$ & $(1.18 \pm 0.61) \mathrm{E}-1$ \\
\hline 080224 & $16: 58: 51.050$ & $(5.40 \pm 0.29) \mathrm{E}+0$ & $1.0 \mathrm{E}-1$ & $(2.22 \pm 0.41) \mathrm{E}-1$ \\
\hline 080318 & 08:31:45.050 & $(1.47 \pm 0.16) \mathrm{E}+1$ & 3.0E-1 & $(1.51 \pm 0.79) \mathrm{E}-1$ \\
\hline 080319 & $12: 25: 56.900$ & $(1.20 \pm 0.06) \mathrm{E}+1$ & $2.0 \mathrm{E}-1$ & $(3.29 \pm 0.42) \mathrm{E}-1$ \\
\hline 080320 & $11: 52: 02.625$ & $(3.05 \pm 0.08) \mathrm{E}+1$ & $2.5 \mathrm{E}-1$ & $(1.96 \pm 0.22) \mathrm{E}-1$ \\
\hline 080328 & 08:03:14.500 & $(8.60 \pm 0.62) \mathrm{E}+1$ & $1.0 \mathrm{E}+0$ & $(3.03 \pm 0.58) \mathrm{E}-1$ \\
\hline 080330 & 11:04:33.450 & $(3.36 \pm 0.09) \mathrm{E}+1$ & 3.0E-1 & $(2.54 \pm 0.20) \mathrm{E}-1$ \\
\hline 080408 & $03: 36: 23.050$ & $(1.40 \pm 0.11) \mathrm{E}+0$ & $1.0 \mathrm{E}-1$ & $(5.29 \pm 0.37) \mathrm{E}-1$ \\
\hline 080413 & $08: 51: 12.250$ & $(7.60 \pm 1.20) \mathrm{E}+0$ & $1.0 \mathrm{E}-1$ & $(0.55 \pm 1.17) \mathrm{E}-1$ \\
\hline 080425 & $20: 21: 47.775$ & $(2.28 \pm 0.32) \mathrm{E}+1$ & $1.5 \mathrm{E}-1$ & $(2.58 \pm 1.05) \mathrm{E}-1$ \\
\hline
\end{tabular}

long GRBs. The most remarkable difference is the hardness of the intermediate class. In the BATSE data, the intermediate class has the lowest hardness ratio, which is anticorrelated with the duration (Horváth et al. 2006), whereas we find for the RHESSI data that its hardness is comparable with that of the short group and correlated with the duration, although this correlation is inconclusive because of its large error. The hardness of the intermediate class found for the RHESSI data is surprising since the intermediate class in the BATSE data was found to be the softest. This discrepancy might by explained by the different definitions of the hardnesses. The hardness $H$ for the RHESSI data is defined as $H=F_{120-1500} / F_{25-120}$, whereas for the BATSE data $H=F_{100-320} / F_{50-100}$, where the numbers denote energy in $\mathrm{keV}$ (the BATSE fluences at higher energies than $320 \mathrm{keV}$ are noisy (Bagoly et al. 1998)). This means that hardnesses do not measure the identical behaviour of bursts. The situation differs even more significantly if we compare hardnesses in the Swift and RHESSI databases, because the Swifts' hardnesses are defined as $H=F_{100-150} / F_{50-100}$ and $H=F_{50-100} / F_{25-50}$ (Horváth et al. 2008; Sakamoto et al. 2008).

The shorter durations of the RHESSI GRBs compared to the BATSE GRBs can be understood in the following way. For RHESSI, which is practically unshielded, the background is high (minimum around 1000 counts per second in the (25-1500) keV band) and varies by up to a factor 3. Additionally, RHESSI's sensitivity declines rapidly below $\approx 50 \mathrm{keV}$. Weak GRBs (in the sense of counts per second) and soft GRBs are not so well observed by RHESSI. Since GRBs tend to be softer and weaker at later times, they should soon fall bellow RHESSI's detection limit, resulting in a shorter duration being inferred.

For Swift, $\approx 58 \%$ of all GRBs are long, $\approx 7 \%$ short and $\approx 35 \%$ intermediate (Horváth et al. 2008). The percentage of each group depends obviously on the used instrument.

\section{Conclusion}

The RHESSI data confirm that GRBs can be separated into short and long classes, and that the short GRBs are on average harder than the long ones. A two-dimensional analysis of the hardness/duration plane as well as a maximum likelihood fit of the duration distribution also indicate a third class with intermediate duration and similar hardness as the short class.

Acknowledgements. This study was supported by the GAUK grant No. 46307, by the OTKA grants No. T48870 and K 77795, by the Grant Agency of the Czech Republic grant No. 205/08/H005, by the Research Program MSM0021620860 of the Ministry of Education of the Czech Republic, by the INTEGRAL PECS Project 98023 and by the grant GA ČR 205/08/1207. We appreciate help of K. Hurley with the RHESSI GRB list, valuable discussion with L.G. Balázs and useful remarks of O. Wigger. Thanks are due to the anonymous referee for the worthwhile notes.

\section{References}

Aptekar, R. L., Butterworth, P. S., Cline, T. L., et al. 1998, AIPC, 428, 10 Band, D. L., Ford, L. A., Matteson, J. L., et al. 1997, ApJ, 485, 747, Appendix A Bagoly, Z., Mészáros, A., Horváth, I., Balázs, L. G., \& Mészáros, P. 1998, ApJ, 498, 342

Chattopadhyay, T., Misra, R., Chattopadhyay, A. K., \& Naskar, M. 2007, ApJ, 667,1017

Freeland, S. L., et al. 2008, http://www . Imsal . com/solarsoft Hakkila, J., Haglin, D. J., Pendleton, G. N., et al. 2000, ApJ, 538, 165 Hakkila, J., Giblin, T. W., Roiger, R. J., et al. 2004, Balt. Astron., 13, 211 Holman, G. D. 2008, http://hesperia.gsfc.nasa.gov/hessi Horváth, I. 1998, ApJ, 508, 757

Horváth, I. 2002, A\&A, 392, 791

Horváth, I., Mészáros, A., Balázs, L. G., \& Bagoly, Z. 2004, Balt. Astron., 13, 217

Horváth, I., Balázs, L. G., Bagoly, Z., Ryde, F., \& Mészáros, A. 2006, A\&A, 447, 23

Horváth, I., Balázs, L. G., Bagoly, Z., \& Veres, P. 2008, A\&A, 489, L1 Hurley, K. 2007, http://www.ssl. berkeley.edu/ipn3/index.html Hurley, K. 2008, http://www.ssl.berkeley.edu/ipn3/masterli.html Kouveliotou, C., Meegan, C. A., Fishman, G. J., et al. 1993, ApJ, 413, 101 Lin, R. P., Dennis, B. R., Hurford, G. J., et al. 2002, Sol. Phys., 210, 3 Meegan, C. A., et al. 2001, Current BATSE Gamma-Ray Burst Catalog, http://gammaray.msfc.nasa.gov/batse/grb/catalog

Mukherjee, S., Feigelson, E. D., Jogesh, B. G. et al. 1998, ApJ, 508, 314

Press, W. H., Feigelson, E. D., Jogesh, B. G., et al. 1992, Numerical Recipes in $\mathrm{C}$ (Cambridge University Press)

RSI IDL, http://rsinc.com/idl/

Sakamoto, T., Barthelmy, S. D., Barbier, L., et al. 2008, ApJS, 175, 179

Smith, D. M., Lin, R. P., Turin, P., et al. 2002, Sol. Phys., 210, 33

Smith, D. M., Lin, R. P., Hurley, K. C., et al. 2003, Proc. SPIE, 4851, 1163

Trumpler, R. J., \& Weaver, H. F. 1953, Statistical Astronomy (Berkeley: University of California Press)

Wigger, C., et al. 2006,

http://grb.web.psi.ch/publications/talk_venice.pdf

Wigger, C., Hajdas, W., Zehnder, A., et al. 2006, Il Nuovo Cimento B, 121, 1117

Wigger, C., et al. 2008, http://grb.web.psi.ch

Zey, C, et al. NIST/SEMATECH, e-Handbook of Statistical Methods, http://www.itl.nist.gov/div898/handbook/ 